Review article

\title{
The harder the climb the better the view: The impact of substrate stiffness on cardiomyocyte fate
}

\author{
Silvia Querceto $^{\text {a }}$, Rosaria Santoro ${ }^{\text {b, c }}$, Aoife Gowran ${ }^{\mathrm{b}}$, Bruno Grandinetti ${ }^{\mathrm{d}}$, Giulio Pompilio ${ }^{\mathrm{b}, \mathrm{e}}$, \\ Michael Regnier ${ }^{\mathrm{f}}$, Chiara Tesi ${ }^{\mathrm{a}}$, Corrado Poggesi ${ }^{\mathrm{a}}$, Cecilia Ferrantini ${ }^{\mathrm{a}}$, Josè Manuel Pioner ${ }^{\mathrm{g}, *}$

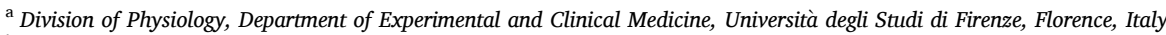 \\ ${ }^{\mathrm{b}}$ Unità di Biologia Vascolare e Medicina Rigenerativa, Centro Cardiologico Monzino IRCCS, via Carlo Parea 4, Milan, Italy \\ ${ }^{\mathrm{c}}$ Department of Electronics, Information and Biomedical Engineering, Politecnico di Milano, Milan, Italy \\ d European Laboratory for Non-Linear Spectroscopy (LENS), Sesto Fiorentino, FI, Italy \\ e Department of Biomedical, Surgical and Dental Sciences, University of Milan, Italy \\ ${ }^{\mathrm{f}}$ Department of Bioengineering, University of Washington, Seattle, WA, USA \\ ${ }^{g}$ Department of Biology, Università degli Studi di Firenze, Florence, Italy
}

\section{A R T I C L E I N F O}

\section{Keywords:}

Substrate stiffness

hiPSC

Cardiomyocytes

Cardiac extracellular matrix

Tissue engineering

Genetic cardiomyopathy

\begin{abstract}
A B S T R A C T
The quest for novel methods to mature human induced pluripotent stem cell-derived cardiomyocytes (hiPSC$\mathrm{CMs}$ ) for cardiac regeneration, modelling and drug testing has emphasized a need to create microenvironments with physiological features. Many studies have reported on how cardiomyocytes sense substrate stiffness and adapt their morphological and functional properties. However, these observations have raised new biological questions and a shared vision to translate it into a tissue or organ context is still elusive. In this review, we will focus on the relevance of substrates mimicking cardiac extracellular matrix (cECM) rigidity for the understanding of the biomechanical crosstalk between the extracellular and intracellular environment. The ability to opportunely modulate these pathways could be a key to regulate in vitro hiPSC-CM maturation. Therefore, both hiPSC$\mathrm{CM}$ models and substrate stiffness appear as intriguing tools for the investigation of cECM-cell interactions. More understanding of these mechanisms may provide novel insights on how cECM affects cardiac cell function in the context of genetic cardiomyopathies.
\end{abstract}

\footnotetext{
Abbreviations: hiPSC-CMs, Human induced pluripotent stem cell-derived cardiomyocytes; cECM, Cardiac extracellular matrix; kPa, Kilo Pascal; CE, Contractile

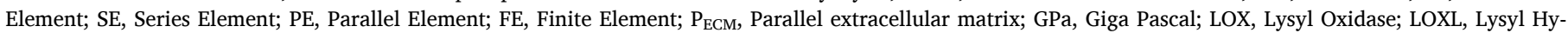

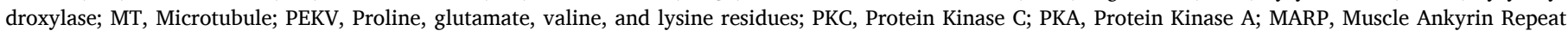

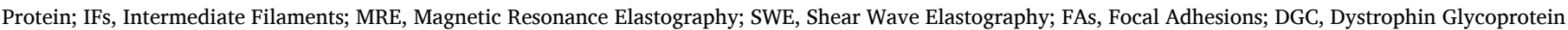

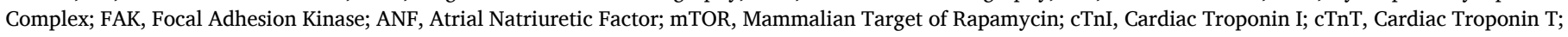

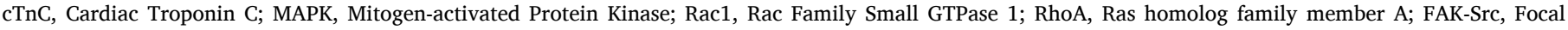

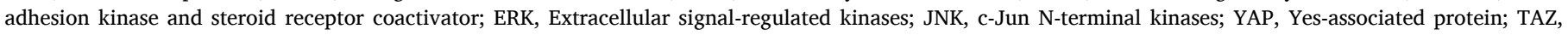

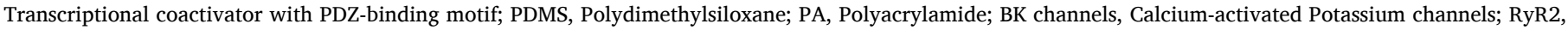

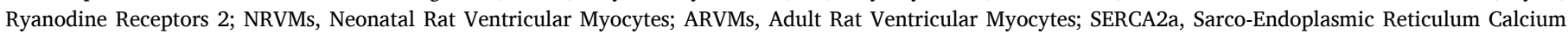

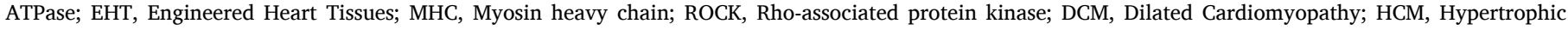

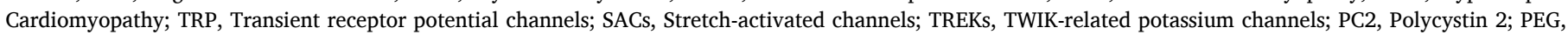

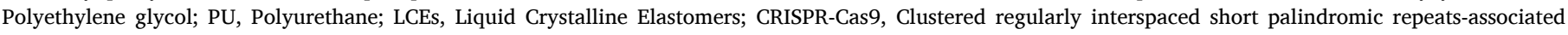

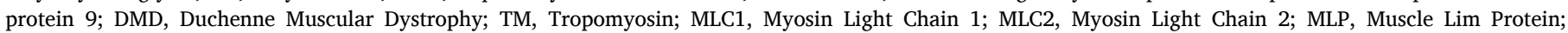

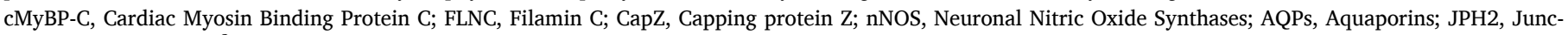
tophilin 2; CaMKII, $\mathrm{Ca}^{2+}$ /calmodulin-dependent protein kinase II.

* Corresponding author.

E-mail address: josemanuel.pioner@unifi.it (J.M. Pioner).
} 


\section{Introduction}

Despite the high potential of cardiomyocytes derived from human induced pluripotent stem cells (hiPSC-CMs) for cardiac disease modelling, drug testing, and heart regeneration, their use is limited by their immature structural and functional features [1]. A simple observation about this problem is related to the current methods for cell culture that are far from recapitulating the physiological tissue conditions, in which chemical and mechanical cues actively orientate cardiac cell fate. The ex vivo reproduction of chemical and mechanical determinants related to cardiac function is challenging as preload and afterload conditions need to be considered in concert with neurohormonal influences. However, the in vitro cardiac microenvironment can be modelled to make the biomimetic substrate as close as possible to the native cardiac extracellular matrix (cECM).

The reproduction of cECM can be designed with a multitude of controlled variants ranging from matrix composition, topography, and other chemo-physical features [2]. In this review, we particularly focus on the impact of substrate stiffness for cardiac cell engineering.

Modulating substrate stiffness plays a crucial role for cell growth and maturation in vivo, as demonstrated through several cell models ranging from non-myocytes (e.g., myotubes, fibroblasts) to embryonic stem cell derived- or neonatal/fetal from human, rodent or avian origin cardiomyocytes. In this context, myocardial elastic modulus ranges from the low kilopascal range (1-6 kPa) in the embryonic heart to approximately $10-15 \mathrm{kPa}$ in the healthy adult heart. Also, the myocardium with elastic moduli beyond $50 \mathrm{kPa}$, which can be caused by increased expression and cross-linking of cECM components, are characteristic of many cardiomyopathies $[3,4]$.

To date, more than 100 published papers have analyzed the interaction of cardiomyocytes with substrate stiffness from a functional, structural, and proteomic perspective (Pubmed search (cardiomyocytes)) AND (substrate stiffness)). Despite this large amount of works, the effects of matrix stiffness on cardiomyocytes remains rather unclear. For this reason, this review focuses on the biophysical and molecular aspects of cardiomyocyte-cECM interaction. The specific aim is to gather together elements that may help to recreate as close as possible the 3D native cardiac microenvironment using combined extracellular matrix proteins or innovative biomaterials. The role of cardiac stiffness determinants and cECM properties is discussed with special attention to the elements involved in the tridimensional tissue scenario. The role of parallel elastic elements (classically described in the Hill's three-element muscle model) are evaluated together with transverse elastic elements represented by the cECM and costameres. Transverse elements are in the spotlight for their role in the adaptiveness of cardiomyocytes to increased substrate stiffness. In conclusion, the relevance of tuning substrate stiffness using in vitro models paves the way of a better understanding of the biomechanical role of cECM that undergoes continuous remodelling during development, aging and cardiac diseases (including genetic cardiomyopathies). Indeed, in pathological conditions it is observed that a progressive increase of tissue stiffness is responsible for a dysfunctional and fibrotic myocardium [5]. Since the study of tissue remodelling and stiffening has limited understanding so far, novel in vitro models that may recapitulate these aspects are of fundamental importance.

\section{Determinants of cardiac tissue stiffness}

To fully understand the importance of cardiac cell and tissue stiffness, the underlying concepts that regulate the elastic properties in response to load and deformations need to be explored. According to Hooke's law, for a linear elastic body that can deform under a certain load (F) with a single degree of freedom (e.g., stretching or compression of a spring), the stiffness is a parameter that describes its resistance to deformation and is defined as:

$$
k=\frac{\mathrm{F}}{\Delta \mathrm{L}}
$$

where, $\mathrm{F}$ is the force applied on the body and $\Delta \mathrm{L}$ is the displacement produced by the force along the same degree of freedom (for instance, the change in length of a stretched spring).

The stiffness is a property of a specific structure (or elastic body), and it depends on its geometrical dimensions and on the material of which it is composed. The characteristics of the latter, described by its modulus, derived from load versus deformation relationship obtained from standardized tests on samples of standardized size and shape. Such tests consider different loading configuration (shear, compression, or elongation) that reflect themselves in different responses of the material upon stress application.

In the case of a homogenous unidimensional bar composed of a single linear elastic material subjected to uniaxial elongation, the intrinsic elastic properties of the material are described by the elastic modulus (or Young's modulus), that can be measured as:

$E=\frac{F / A}{\Delta L / L_{0}}=\frac{F L_{0}}{A \Delta L}$

where $\mathrm{E}$ is bar elastic modulus, $\mathrm{F}$ is the applied force for deforming the body, $\Delta \mathrm{L}$ is the displacement of the application point of the force and $\mathrm{L}_{0}$ is bar initial length. Rearranging the terms, the relation $\mathrm{F}=\mathrm{k} \Delta \mathrm{L}$ holds, and $\mathrm{k}=\mathrm{AE} / \mathrm{L}$ is bar axial stiffness. Under those assumptions, an analogy between Hooke's law for force-displacement relationship and the stressstrain curve in solid deformation can be made, and the elastic modulus is reflected in the stiffness of the final structure. Thus, when describing the stiffness of two linear isotropic elastic materials, the higher the elastic modulus, the stiffer the material (considering the same uniaxial stretching and geometrical constraints). This law can be applied for Hookean solids and provides a general idea of the concept underlying spring-like linear elasticity of materials.

In biological tissues the scenario is more complicated. Firstly, any biological tissue is a composite material whose individual components have specific and rather disparate elastic moduli. In addition, while biological tissues can be prepared as individual bodies of specific size and shape (e.g., muscle bundles or strips) for experimental purposes, in the organ context the 3D force vector orientation is complex and almost impossible to be replicated ex vivo. Thus, natural materials do not usually display linear stress-strain relationship and could exhibit a dependency of stress on strain rate. To further complicate the scenario, the intrinsic directionality of the majority of synthetic and biological materials should be considered. The anisotropy of a material allows it to change or assume different properties in different directions (Fig. 1A). In the case of elastic bodies constituted by anisotropic materials, the elastic moduli can be completely different if the forces are applied longitudinally or transversely to its preferential axis. By looking at the striated muscle structure, we can identify different components with different active and passive properties, respectively [6]. The Hill's three-element muscle model is a representation of the muscle mechanical response. It considers a contractile element (CE) and an elastic element in series (SE) to describe the active unit of the fiber and another elastic element in parallel (PE) to the former to model its passive properties.

This model, although powerful, fails to completely outline the behaviour of an entire muscle, as it does not consider passive contributions of transverse elements, such as ECM. For instance, Marcucci and coworkers provided an extension of Hill's model, describing ECM contribution as an elastic element in parallel to muscle fiber (Fig. 1B). In the context of a finite element (FE) model of a muscle, separated meshes for the muscle and the ECM can better describe intra- (PE, red finite element regions) and extra- ( $\mathrm{P}_{\mathrm{ECM}}$, blue finite element regions) sarcomeric components of the passive forces in muscle bundles [6].

The phenomenological description of striated muscle behaviour through Hill-type three-element model have been extended to a two- 
A

B
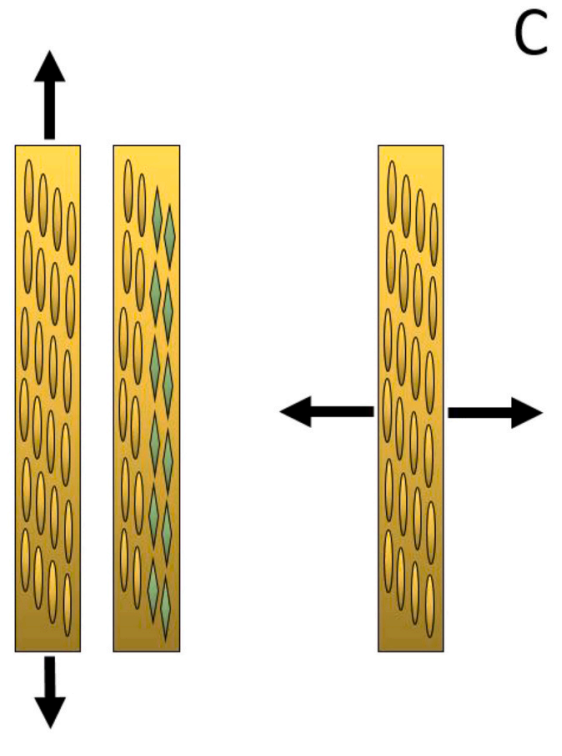

C

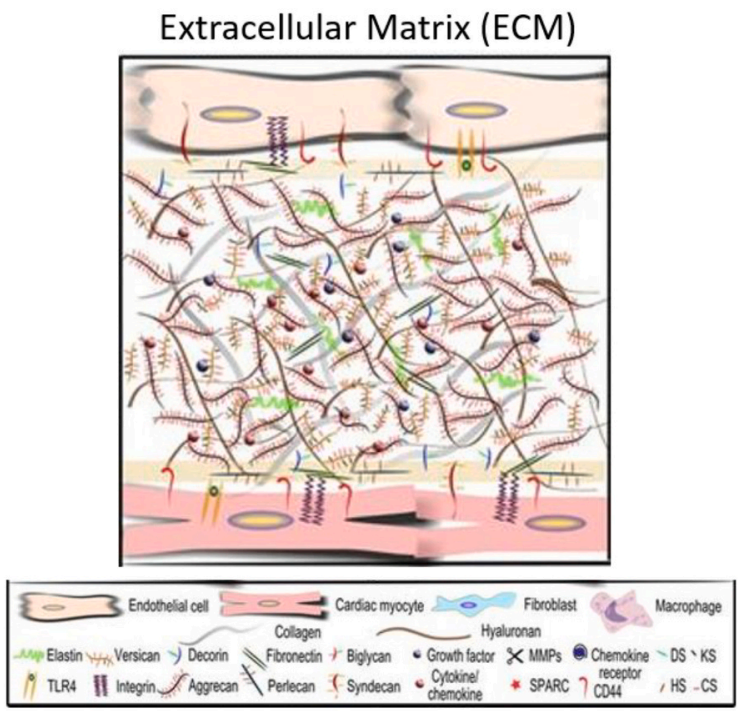

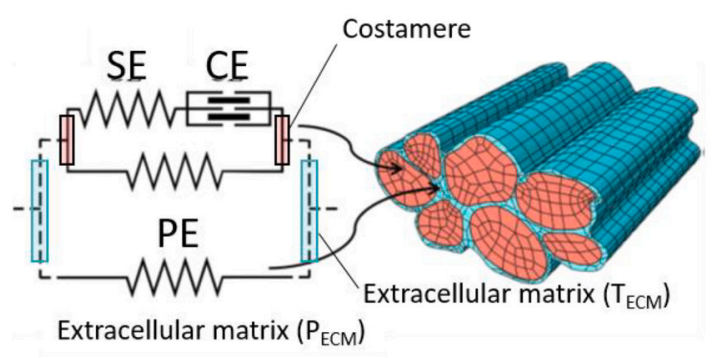

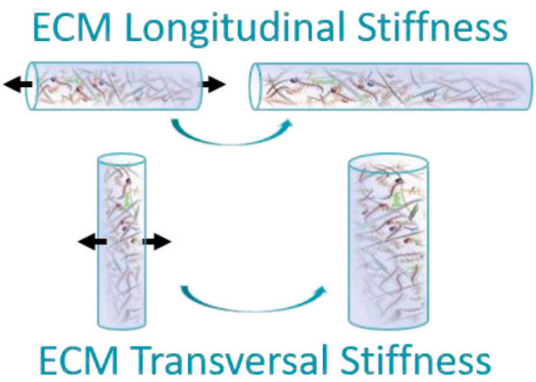

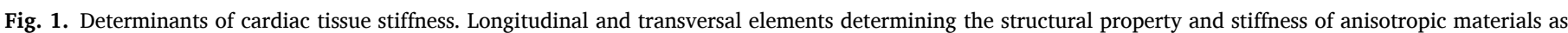

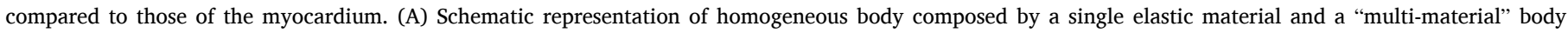

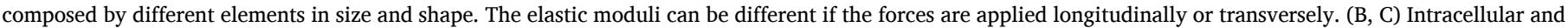

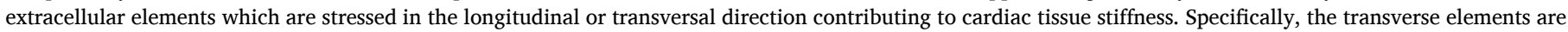

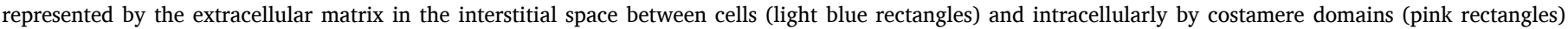

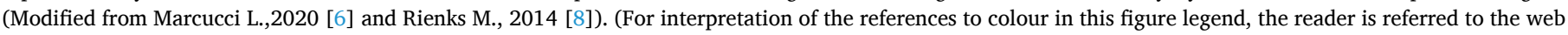
version of this article.)

and three- dimensional scale [7], well defining the components that are stressed in the longitudinal direction (i.e., the force vector given by the contractile apparatus).

However, less attention was given to the transversal elements (Fig. 1B) often simplified as points of application of the forces involved in the contractile process. Indeed, these structures are elastic elements that are stressed, though, in a transverse direction or produced bending or twisting movements.

Specifically, the transverse elements are represented intracellularly by costameres and by the ECM in the interstitial space (Fig. 1C) [8].

\section{1. cECM stiffness}

The cECM provides not only structural support, but it has a much broader foundational role by regulating numerous cellular functions, such as proliferation, migration, metabolism, survival and differentiation [8]. In the myocardium, cardiomyocytes occupy about $75 \%$ of the entire volume and are organized as thin laminae of 2-5 cell layers [5]. The surrounding cECM is mainly composed by fibrillary collagen and elastin proteins and it does not have only passive mechanical properties, but it is also functionally involved in tissue development, injury, and disease remodelling, owing to the presence of resident cells such as fibroblasts and macrophages (Fig. 1C). Cardiac fibroblasts, the main matrix-producing cells, are the largest cell population in normal mammalian hearts and are typically enmeshed in the endomysium and perimysium [9].

Collagen is the most abundant structural $\mathrm{CECM}$ protein and provides structural support and tensile strength. In the cECM, type I collagen represents $75-80 \%$ of the protein content whereas type III collagen represents approximately $15-20 \%$ of the total collagen isoforms. In the rat myocardium, both collagen I and III expression rises throughout the fetal, neonatal, and adult stages [10]. Collagen I expression can dramatically change during aging or injured myocardium [11]. Interestingly, collagen IV is the only collagen secreted by cardiomyocytes and is mainly collected in the basal membrane in association with laminin and fibronectin [12,13]. In human adult ventricular myocardium, collagen IV, laminin and fibronectin span the inner lining of the Ttubular network and are thought to prevent the collapse of T-tubules during cardiac contraction $[12,14,15]$. Also, the regulation of collagen composition is an important feature of valve formation [16]. A potential role of type $\mathrm{VI}$ collagen in atrioventricular (AV) valve morphogenesis is supported by its expression during the developing myocardium [17]. In human myocardium, collagen VI is located in the endomysium and perimysium surrounding blood vessels and individual myocytes and enlarge its distribution in failing myocardium [18]. Indeed, type VI collagen expression is first commensurate with cell migration within the $\mathrm{AV}$ cushions and then it forms an extracellular network within the valve leaflets [19]. Overall, 28 collagen isoforms are currently described and, considering both single molecules and fibrils, collagen elastic modulus was estimated in the Giga Pascal (GPa) range [20]. For instance, in the 
mammalian tendon, collagen elastic modulus ( $90 \%$ of resilience) is approximately $1.2 \mathrm{GPa}$. In contrast, in the porcine aorta elastin elastic modulus ( $77 \%$ of resilience) is about $0.81 \mathrm{MPa}$. The process of collagen crosslinking can be initiated by enzymatic and non-enzymatic reactions [21]. For instance, lysyl oxidases (LOX) as well as LOX-like proteins (LOXL-1 and LOXL-2) and lysyl hydroxylases are some of the wellknown enzymes that trigger collagen crosslinking [22]. It has been demonstrated that collagen crosslinking contributes to the passive tension of rat trabeculae at physiological sarcomere lengths in the heart (1.9-2.2 $\mu \mathrm{m})$, and even more at longer sarcomere lengths [23]. Increased cardiac passive stiffness is associated with diastolic dysfunction. Notably, increased LOX expression has been reported in patients with myocardial fibrosis and correlates with enhanced passive stiffness as well as diastolic dysfunction due to excessive collagen crosslinking [24,25].

The other important fibrillar ECM molecule is elastin, whose name accounts for the intrinsic elastic properties. Elastin contributes to tissue flexibility and elasticity by reducing the elastic modulus and making a tissue structure more compliant. The elastic modulus of elastin fibers is approximately 1.1-1.2 MPa [20].

Under intrinsic or extrinsic-induced injuries, activated fibroblasts are the crux for myocardial fibrosis. Conversion of fibroblasts into secretory, matrix-producing and contractile cells, called myofibroblasts, is a crucial cellular event in many fibrotic conditions, as widely reviewed by Frangogiannis [5].

\subsection{Cell stiffness}

The major intracellular contributors to passive stiffness are titin, intermediate filaments, and the microtubule (MT) network [23]. Titin is the largest human protein that spans from the $Z$-disc to the M-band of the sarcomere. The I- and A-band domains impart the major biophysical properties of titin [26,27]. The I-band is responsible for the elastic behaviour of titin and accountable for the passive elasticity of cardiac muscle. The A-band is an inextensible domain rigidly attached to the myosin filament with no or minimal possibility for elongation. In the Iband, titin consists of two immunoglobulin-like (Ig) domains (distal and proximal) and the spring-like domains N2A and PEVK-element [28]. These four spring elements react in different ways under a stretching force, suggesting that these regions have different mechanical properties. When force is applied, the distal (Ig) domains stretch through an unfolding intermediate state. This unfolding intermediate state provides increased mechanical resistance [28,29].

Studies performed in rat cardiac muscle have shown that at similar physiological sarcomere lengths, cardiac titin is able to develop much higher passive tensions than in skeletal muscle [23]. Notably, in contrast to the skeletal isoform, the titin isoform expressed in rat cardiac muscle has a lower molecular mass and it is characterized by a much shorter flexible I-band domain. Therefore, the extensible I-segment of cardiac titin can be strained to a higher degree at any given sarcomere lengths, and this is the main support for the idea that cardiac titin is able to develop higher tensions at shorter sarcomere length [30,31]. Several proteins interact.

with the titin-N2A element, such as SMYD2, P94/calpain3, and the muscle ankyrin repeat proteins (MARPs). Titin-N2A might also interact with the actin-based thin filament, presumably in a $\mathrm{Ca}^{2+}$-dependent manner [32]. Recent findings in skeletal muscle identified MARPinduced locking of titin-N2A to actin and the resulting increase in passive force might be a mechanism to stabilize sarcomeres and protect them from mechanical stress. Compared with skeletal muscles, the role of the locking mechanism might be more finely tuned in the heart [33]. During heart development, titin's elasticity is flexible due to expression of different I-band components, including the N2B and PEKV spring, which are differently expressed in human heart [34-36]. Interestingly, titin elasticity can be modulated by mRNA splicing events that generate isoforms of different size and protein phosphorylation by calcium binding kinases such as PKC and PKA [27,37]. Overall, collagen and titin can be considered the most important elements in determining cardiac passive stiffness and tension, with titin dominating at shorter lengths of the working range of sarcomeres in the heart. In summary, titin plays a crucial role in the functioning of striated muscles, and it is a main contributor to passive tension $[23,27,38]$. The elastic nature of the Iband and the wide range of extensibility depending on force applied contribute to length-dependent activation described in the FrankStarling curve [39].

In addition to titin, the extra-sarcomeric cytoskeleton, including the MT network and intermediate filaments (IFs), contributes to the passive mechanical properties of cardiomyocytes. Owing to their viscoelastic properties, microtubules counteract deforming stress, and this event is particularly enhanced through crosslinking with actin and intermediate filaments to impose resistive intracellular load on the shortening sarcomere [40]. It has been demonstrated that this event mainly depends on a specific post-translational modification of microtubules, namely detyrosination initiated by tubulin carboxypeptidase (TCP), which leads to crosslinking with the protein desmin at $Z$-discs and causes high-resistance during cardiomyocyte contraction [41,42]. However, the role of microtubules is still under debate. High resolution imaging of microtubules during cardiomyocyte contraction revealed their deformation under contractile load into sinusoidal buckles and this event was found to be dependent on the detyrosination of $\alpha$-tubulin. Detyrosinated MTs associated with desmin are more tightly anchored to sarcomeres leading to reduced force-generation [41]. Lastly, intermediate filaments in cardiac cells mainly consist of desmin, a muscle-specific IFs protein, that is highly expressed in the myocardium compared to skeletal muscle. It has been reported that IFs contribute to passive stiffness especially at shorter sarcomere lengths between 1.9 and $2.1 \mu \mathrm{m}$ in the heart with a declining contribution at longer lengths [23].

Limited understanding of the relative contribution of CECM and intracellular elastic elements to passive properties of cardiac muscle is a major obstacle to unravel the molecular mechanisms of subcellular structures. Overall, intracellular and extracellular determinants act as elastic elements in parallel to the active contractile elements (actin and myosin). Passive biophysical properties of cardiac muscle/myocytes have been primarily assessed along the longitudinal direction. Instead, the compression of transverse elastic elements has been less investigated and may play a major role in the interaction between cells and cECM. For instance, tension/compression asymmetry was observed in skeletal muscle. A comparison between compressive and tensile tests by Takaza et al. indicated that the stress-strain response in tension is always around two orders of magnitude greater than in compression [43]. Therefore, a clear comprehension of the role of elastic elements is relevant for the design of an in vitro microenvironment mimicking the physiological condition, as the engineered biomaterials need to have mechanical properties that match those of the replaced native tissue.

\subsection{Developmental changes of cardiac stiffness}

Adding further complexity, the extracellular and intracellular components undergo spatial and temporal changes during cardiac development. Throughout the lifespan from embryonic to adult stage [44], the architecture and the composition of cECM undergo continuous cellmediated turnover, ensuring the dynamic remodelling of the $\mathrm{CECM}$ niche $[5,11,14,45]$. It is well known that cardiac fibroblasts are the main source of depositing proteins within the cECM to form a network around cells that reinforces cardiac architecture. The way in which cardiac fibroblasts sense and modify the cECM has widely been reported. At cellular level, the fetal heart is characterized by dramatic changes in the isoform pattern of many sarcomeric proteins $[46,47]$. In particular, embryonic isoform switching includes titin [47,48]. For instance, embryonic titin exists in the prenatal heart as a unique, compliant (long spring), large size N2BA-isoform. Before or around birth, this fetal isoform is replaced by a smaller, less extensible, N2BA-isoform co- 
expressed with a stiffer N2B-isoform, which is most abundant in the postnatal hearts (left ventricles) of many mammalian species [46]. Generally, the sarcomeres of fetal or neonatal hearts are much more compliant than those of adults [49]. For instance, the elastic modulus of individual human embryonic stem cells (hESCs) and hESC-derived cardiomyocytes are reported as 5 and 15 Pa respectively as estimated by optical tweezer technique [50]. Together with increasing stiffness, the myocardium undergoes significant remodelling during development leading to faster beating frequency, elevated end-diastolic pressure, stroke volume, left ventricular (LV) dimensions and increased ejection fraction. Furthermore, as confirmed by in vivo measurements using cardiac magnetic resonance elastography (MRE) and ultrasound cardiac shear-wave elastography (SWE), cardiac stiffness was observed to vary between individuals and animal species as well as between different regions of the heart [51,52].

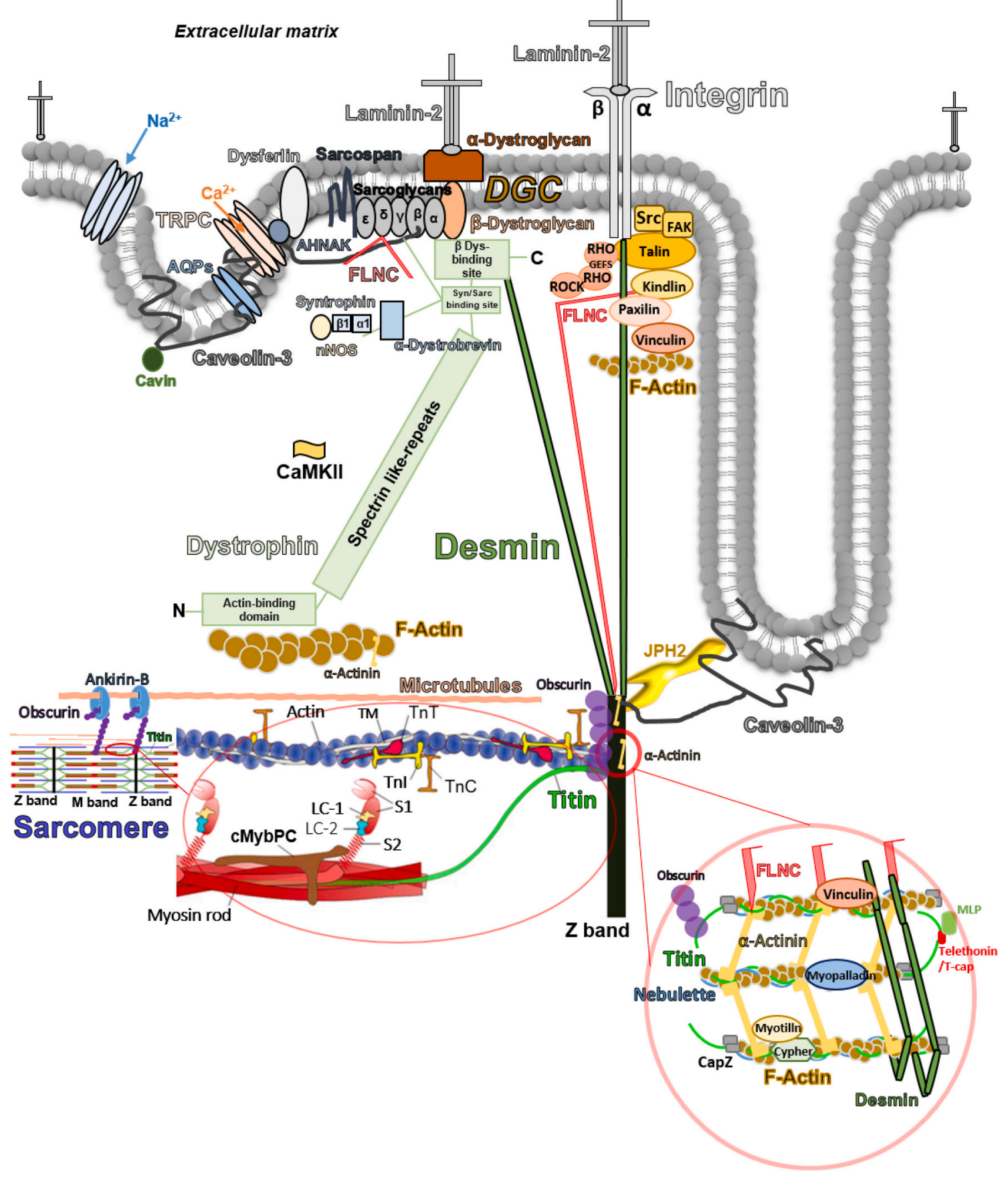

Fig. 2. Cardiac ECM-cell interaction. Costameres are subsarcolemmal, $Z$-lines associated structures found in striated muscle and composed of proteins that are involved in the CECM-cell interaction. The dystrophin-glycoprotein complex (DGC) is one of the major protein complexes associated with Z-disc structures. Dystrophin, sarcoglycans, sarcospan, dystroglycans and syntrophins constitute core components of the DGC. Dystrophin binds both actin and $\beta$-dystroglycan, connecting the transmembrane components of the DGC to the cytoskeleton. The second complex present at the cardiac costameres is the integrin-vinculin-talin complex. Integrins are heterodimeric transmembrane receptors which once bound to their specific cECM ligands, are responsible for the formation of focal adhesions (FAs) that link the cECM to the cytoskeleton. 


\section{3. cECM-cell interaction: the costamere}

The functional role of the ECM in transmitting passive tension at the whole muscle level is still unclear. Heart functionality is tightly linked to a complex crosstalk based on chemical, electrical and mechanical coupling between cells. The processes of mechanosensing and mechanotransduction can be defined as the capacity of cells to feel the mechanical properties of the surrounding environment and to actively adapt to its modifications [53]. Mechanosensitive proteins are located both at cell-cell and cell-matrix adhesions [44]. Cell-matrix adhesions anchor maturing cells to the ECM, direct cell migration and morphogenesis, and they are facilitated by cell surface receptors, such as integrins and cadherins. In particular, the mechanical properties of ECM induce an adaptive response within the cytoplasm and the nucleus, leading to changes in cell fate specification and function [53]. Transverse lattice elements called costameres are involved in the cell-cECM interaction that was first described in the skeletal muscle in the early 1980s [54] (Fig. 2). Costameres are subsarcolemmal protein assemblies that circumferentially align with the $Z$-disks of peripheral myofibrils and physically couple force-generating sarcomeres with the sarcolemma in striated muscle cells [55]. The costamere has garnered renewed attention because several of its constituent proteins are defective in the mechanosensing and mechanotransduction in muscular dystrophies and cardiomyopathies [56].

The development of active force by myosin motors pulling on actin filaments generates substantial mechanical stress on various structures of the sarcomere, which needs to be counteracted and dissipated through cytoskeletal proteins to cECM or adjacent cells. Indeed, this traction force does not solely involve axial stiffness provided by sarcomere elastic proteins (e.g., titin) but also transverse stiffness provided by Z-disk and M-band proteins [57]. Both Z-disks and M-bands are implicated in active signalling processes relaying information on active or passive strain to cellular systems that control gene expression, protein synthesis, and protein degradation [58]. Simultaneously, active forces generated by myosin motors are passively transmitted also transversally via integrin and dystrophin complexes to the ECM [6], thanks to a network of cytoskeletal and membrane proteins that operate in concert to exert force on the cECM through focal adhesions (FAs). From the mechanotransduction view point, the $Z$-disk is not only responsible for transmitting force between sarcomeres during cardiac contraction, but it also represents a hot-spot for cellular signalling [59]. Indeed, Z-disks integrate signals from two main sub-membranous Z-disk-associated structures that form large protein complexes, namely dystrophinglycoprotein complex (DGC) and integrin-vinculin-talin complex. The main function of the sarcolemma-associated proteins of the DGC is to provide a physical linkage across the muscle sarcolemma to connect the cECM with the F-actin cytoskeleton. The proteins classified as core components of the DGC include dystrophin, sarcoglycans, sarcospan, dystroglycans and syntrophins. Laminin in the ECM binds $\alpha$-dystroglycan, a membrane protein also interacting with $\beta$-dystroglycan, that is stabilized at the sarcolemma by the sarcoglycan-sarcospan complex. Dystrophin binds both actin and $\beta$-dystroglycan, connecting the transmembrane components of the DGC to the cytoskeleton. Therefore, dystrophin and DGC deficiency impairs striated muscle integrity and leads to contraction-induced damage $[59,60]$. Notably, mutations in the genes encoding many proteins are strictly related to dilated cardiomyopathy [60]. The second complex present at cardiac muscle costameres is the integrin-vinculin-talin complex $[11,15,61]$. Integrins are heterodimeric transmembrane receptors composed of $\alpha$ and $\beta$ subunits which, once bound to their specific ECM ligands, form focal adhesions (FAs) and effectively link the ECM to the cytoskeleton. Integrins can transmit signals from the ECM to the cytoplasm and nucleus (outside-in), leading to an intracellular reaction that affects cell behaviour and the composition of ECM (inside-out) [61]. The extracellular domains of integrins interact with ECM ligands while the cytoplasmic tails interact with actin associated adaptor proteins, such as talin, vinculin and kindlin. The expression of integrin types and isoforms undergo changes during cardiac development and are modified as an adaptive response to cardiac disease. In early cardiac muscle development, cardiomyocytes express $\alpha 5$ and $\alpha 6$ integrin subunits dimerized with both $\beta 1 \mathrm{~A}$ and $\beta 1 \mathrm{D}$ (mouse) [62]. The $\alpha 5$ integrin subunit is replaced by $\alpha 7$ in adult CMs (mouse) [63]. At birth, integrin subunit expression undergoes a switch and $\alpha 7 \mathrm{~B} \beta 1 \mathrm{D}$ integrin becomes dominantly expressed (mouse) [62]. Specific cardiac integrins (e.g., $\alpha 5-\beta 1, \alpha v-\beta 3$ ) are crucial for the heart's response to stress. The response of cardiomyocytes to mechanical stretch has shown to be mediated by $\beta 1$ integrin and focal adhesion kinase (FAK) [61]. Specifically, mechanical stretch in rat ventricular cardiomyocytes enhances FAK phosphorylation and its migration from the perinuclear region to myofilaments leading to the expression of the atrial natriuretic factor (ANF) gene [64]. Additionally, cyclical mechanical stretch was found to promote mTOR-dependent proliferation and pro-fibrotic activation of rat cardiac fibroblasts leading to stress-induced adverse cECM remodelling and resulting in heart failure $[65,66]$. The relevance of $\beta 1$ integrin in guiding in vitro cardiomyocyte maturation was demonstrated by the neutralization of integrin $\beta 1$ receptors via blocking antibodies and pharmacological blockade of FAK. The maturation process of human pluripotent stem cell derived cardiomyocytes was impaired as indicated by a lack of structural cell maturation and reduced expression of mature myofilament markers e.g., cardiac Troponin I (cTnI) [67].

The major chemical signals elicited by mechanical stress at the cell surface are calcium influx through cation channels, stimulation of mitogen-activated protein kinases (MAPK) and changes in the activity of small GTPases such as Rho, Rac1 and RhoA [61]. In particular, the last two cascades are strongly involved in the integrin-mediated mechanotransduction. Rac1, whose activation relies on FAK-Src signalling pathway, controls actin polymerization and the formation of new focal complexes at the edge of the cells, while RhoA is necessary for stress fiber formation $[61,68]$. Additionally, $\beta 1$ integrin promotes the phosphorylation of intracellular MAP kinases such as ERK, JNK and p38. Through these mechanisms, cells sense the stiffness of their surroundings, form FAs, remodel the cytoskeleton, and activate transduction cascades [69]. Mechanosensing of matrix rigidity has been linked to two nuclear transcription factors: Yorkie homologous Yes-associated protein (YAP) and transcriptional coactivator with PDZ-binding motif (TAZ) which are the terminal contributors of the hippo pathway [53,70]. Their involvement was demonstrated in heart development, cardiomyocyte apoptosis after myocardial infarction, and hypertrophic as well as dilated cardiomyopathies $[44,71,72]$. The function of these two factors is required to convey the effects of a stiff ECM while inactivation of YAP/ TAZ is associated with a soft ECM [53,73]. In addition, the expression of several cardiac genes is affected by the stiffness of the extracellular environment. This is confirmed by the upregulation of alpha actinin and myosin heavy chain in neonatal murine cardiomyocytes cultured on softer rather than stiffer substrates. On the other hand, the expression of cardiac immature genes (Nkx-2.5) and proteins (GATA-4) is evident in cardiac cells grown on stiffer substrates [74].

Continuing advances in the identification and characterization of transverse intracellular elements, i.e., $Z$-line and/or costameres involved in several form of genetic cardiomyopathies, highlights the importance of signal transmission between the sarcomere, sarcolemma and cECM.

\section{How substrate stiffness affects cardiomyocytes}

The contribution of all these mechanisms is difficult to dissect in the complex tissue scenario. Instead, the simplified in vitro model is suitable for the understanding of fine molecular mechanisms of cECM-cell interactions. In particular, it has been observed that cardiomyocytes are able to sense the stiffness of the substrate [75] on which they grow. In Table 1, we report a collection of studies in which substrate stiffness is modulated with the use of synthetic materials or native proteins and followed by cardiomyocyte structure and function assessment. 
Table 1

The impact of substrate stiffness on morphological and functional properties of in vitro cardiac models.

\begin{tabular}{|c|c|c|c|c|}
\hline $\begin{array}{l}\text { Young's Moduli } \\
\text { (kPa) }\end{array}$ & Biomaterials & In-vitro model & Parameter assessed & Effect \\
\hline $\begin{array}{l}0.47,11.13 \\
21.51\end{array}$ & Collagen I-based EHTs [88] & hiPSC-CMs & $\begin{array}{l}\text { - mechanical output } \\
\text { - cell morphology } \\
\text {-EC coupling }\end{array}$ & $\begin{array}{l}\text { - enhanced force generation with increased static } \\
\text { stress } \\
\text { - greater cell alignment and size } \\
\text { - increased calcium handling protein expression }\end{array}$ \\
\hline $1-25$ & PA substrates [80] & NRVMs & $\begin{array}{l}\text { - AP dynamics } \\
\text { - L-type } \mathrm{Ca}^{2+} \text { transient }\end{array}$ & $\begin{array}{l}\text { - increased AP duration on gels with } 9 \mathrm{kPa} \\
\text { - greatest inward L-type } \mathrm{Ca}^{2+} \text { current on gels } \\
\text { with physiological stiffness }\end{array}$ \\
\hline $1,5,10,25,50$ & Collagen I-coated PA gels [76] & NRVMs & $\begin{array}{l}\text { - cell morphology } \\
\text { - calcium handling } \\
\text { - contractility }\end{array}$ & $\begin{array}{l}\text { - well aligned striations on } 10 \mathrm{kPa} \\
\text { - larger calcium transients on } 10 \mathrm{kPa} \text { gels } \\
\text { - greater mechanical force on } 10 \mathrm{kPa} \text { gels }\end{array}$ \\
\hline $1,11,34$ & Collagen I-coated PA gels [83] & $\begin{array}{l}\text { Embryonic chick } \\
\text { cardiomyocytes }\end{array}$ & $\begin{array}{l}\text { - cell morphology } \\
\text { - mechanical output }\end{array}$ & $\begin{array}{l}\text { - enhanced striated cytoskeleton organization } \\
\text { - increased contractile work on gels with heart- } \\
\text { like elasticity }\end{array}$ \\
\hline $1,18,50$ & Laminin-coated PA gels [79] & $\begin{array}{l}\text { Embryonic chick } \\
\text { cardiomyocytes }\end{array}$ & $\begin{array}{l}\text { - beating rate } \\
\text { - force contraction } \\
\text { - cytoskeleton structure }\end{array}$ & $\begin{array}{l}\text { - highest beating rate on } 18 \mathrm{kPa} \text { gels during the } \\
\text { first days } \\
\text { - larger forces, more aligned sarcomeric } \\
\text { - striations and higher number of FAs on stiffer } \\
\text { gels }\end{array}$ \\
\hline $2,9,16$ & Gelatin 3D hydrogels [106] & hiPSC-CMs & $\begin{array}{l}\text { - contractility } \\
\text { - sarcomere structure }\end{array}$ & $\begin{array}{l}\text { - increased force generation with muscle- } \\
\text { mimicking stiffness } \\
\text { - enhanced sarcomere organization }\end{array}$ \\
\hline $3.1,4,16$ & acrilammide/bisacrilammide [87] & hiPSC-CMs & $\begin{array}{l}\text {-sarcomere structure } \\
\text {-SERCA localization } \\
\text {-calcium handling } \\
\text {-pathway }\end{array}$ & $\begin{array}{l}\text {-enhanced sarcomere organization } \\
\text { - Increased SERCA2 expression } \\
\text { - Improvement in calcium cycling } \\
\text { - Activation of Rho/ROCK pathway }\end{array}$ \\
\hline $3,8,10,15$ & PDMS array of microposts [77] & NRVMs & $\begin{array}{l}\text { - twitch power } \\
\text { - calcium concentration } \\
\text { - myofibril morphology }\end{array}$ & $\begin{array}{l}\text { - greater twitch power on stiffer posts } \\
\text { - enhanced intracellular calcium levels } \\
\text { - improved myofibril structure }\end{array}$ \\
\hline $3-144$ & Collagen I-coated PA gels [82] & NRVMs & $\begin{array}{l}\text { - cell morphology } \\
\text { - contractility }\end{array}$ & $\begin{array}{l}\text { - optimal cell morphology on } 22-50 \mathrm{kPa} \text { gels } \\
\text { - development on gels with stiffness comparable } \\
\text { to the native rat adult myocardium }\end{array}$ \\
\hline $4-101$ & Gelatin-coated PA gels [91] & hiPSC-CMs & $\begin{array}{l}\text { - force contraction } \\
\text { - sarcomere shortening }\end{array}$ & $\begin{array}{l}\text { - increased force contraction up to } 90 \mathrm{kPa} \\
\text { - sarcomere shortening does not change at } \\
\text { supraphysiological stiffness }\end{array}$ \\
\hline $5-101$ & PDMS gels [78] & hiPSC-CMs & $\begin{array}{l}\text { - cell morphology } \\
\text { - calcium handling }\end{array}$ & $\begin{array}{l}\text { - enhanced morphological and functional } \\
\text { properties } \\
\text { on } 21 \mathrm{kPa} \text { substrates }\end{array}$ \\
\hline $8,15,50,100$ & PA gels [98] & ARVMs & $\begin{array}{l}\text { - cell contraction } \\
-\mathrm{Ca}^{2+} \text { signalling }\end{array}$ & $\begin{array}{l}\text { - impaired cell shortening by stiff cECM and } \\
\text { reduced kinetics } \\
\text { - impaired calcium } \\
\text { Cycling }\end{array}$ \\
\hline 10 & Matrigel-coated PA gels [81] & hiPSC-CMs & $\begin{array}{l}\text { - sarcomere activity } \\
\text { - mechanical output }\end{array}$ & $\begin{array}{l}\text { - increased myofibril alignment and movement } \\
\text { on } 10 \mathrm{kPa} \text { hydrogels } \\
\text { - improved contractility }\end{array}$ \\
\hline $10,100,400$ & PDMS and PA gels [128] & NRVMs & $\begin{array}{l}\text { - cell morphology } \\
\text { - signalling pathways }\end{array}$ & $\begin{array}{l}\text { - increased cell size with substrate stiffness } \\
\text { - stiffer substrates promote signalling pathways } \\
\text { related to cardiac hypertrophy }\end{array}$ \\
\hline 46,457 & Poly-D-lysine-coated PDMS substrates [86] & $\begin{array}{l}\text { Embryonic chick } \\
\text { Cardiomyocytes }\end{array}$ & - electrophysiology & $\begin{array}{l}\text { - stiff substrate upregulates } \mathrm{L}^{-\mathrm{Ca}^{2+}} \text { channel } \\
\text { currents } \\
\text { - stiff substrate inhibits the BK channel function }\end{array}$ \\
\hline $\begin{array}{l}90,450,1200 \\
9200\end{array}$ & PDMS posts (EHTs) [85] & hiPSC-CMs & $\begin{array}{l}\text { - cell maturation } \\
\text { - force contraction }\end{array}$ & $\begin{array}{l}\text { - moderate afterload improved maturation of } \\
\text { hiPSC-CMs } \\
\text { - the power and the work of EHTs are maximal } \\
\text { under } \\
\text { intermediate afterload }\end{array}$ \\
\hline $1 \times 10^{6}$ & $\begin{array}{l}\text { cardiomyocytes in floating cardiac bodies } \\
\text { (CB) vs. culture on glass coverslips [93] }\end{array}$ & hESC-CMs & $\begin{array}{l}\text { - } \beta \text {-MyHC expression } \\
\text { - CMs contractility and } \\
\text { calcium transients }\end{array}$ & $\begin{array}{l}\text { - switch from } \alpha \text { - to } \beta \text {-MyHC in CMs plated on } \\
\text { glass but not in CB cultures } \\
\text { - faster twitch contraction and calcium transients } \\
\text { for CMs on glass coverslips }\end{array}$ \\
\hline $1000-133,000$ & Poly- $\varepsilon$-caprolactone planar layers [74] & $\begin{array}{l}\text { Neonatal murine } \\
\text { cardiomyocytes }\end{array}$ & $\begin{array}{l}\text { - cardiac gene expression } \\
\text { - electromechanical coupling }\end{array}$ & $\begin{array}{l}\text { - expression of immature cardiac genes and } \\
\text { proteins on stiffer substrates } \\
\text { - more organized electromechanical coupling on } \\
\text { softer layers }\end{array}$ \\
\hline
\end{tabular}

\subsection{Structure}

Myocardium rigidity has been in vitro mimicked by using polymers of easy tunable stiffness, such as polydimethylsiloxane (PDMS) or polyacrylamide (PA) [76-80]. These naturally inert substrates can be coated by ECM components (e.g., vitronectin, collagen, fibronectin) to provide ligands for cardiomyocyte integrins. Specifically, the range of elastic moduli representative of the healthy myocardium lies between approximately $10 \mathrm{kPa}$ and $30 \mathrm{kPa}$. When cultured on substrates within this range of stiffness, embryonic or neonatal cardiomyocytes and hiPSC-CMs can acquire, retain, or regain a physiological morphology characterized by a highly elongated anisotropic shape with aligned myofibrils. Indeed, mathematical descriptions of cell shape and quantification of connexin 43 protein demonstrated that cardiomyocytes are more elongated and display correct electromechanical connections when cultured on substrates with physiological stiffness compared to 
those grown on softer $(<10 \mathrm{kPa})$ surfaces upon which cells develop a rounded morphology with disperse actin filaments [76,77,79,81-83] (Table 1). Substrates with elastic moduli in the range of those of the healthy tissue support the structural maturation of cardiomyocytes by promoting increased cell growth in the longitudinal direction, myofibril alignment and bundling and the development of more organized sarcomeres [84]. Specifically, Rodriguez et al. [77] demonstrated that rat neonatal cardiomyocytes on fibronectin-coated microposts with elastic moduli in the physiological range showed a higher sarcomere length and $Z$-band width, suggesting that myofibril structure is affected by substrate stiffness and a biomechanical stimulation can lead to increased contractility.

\subsection{Calcium handling}

Several studies have demonstrated that substrate stiffness not only affects morphological but also functional properties of cardiomyocytes [85]. Contractile force of cardiomyocytes is modulated by the size and duration of the calcium transient, which mainly depends on the dynamics of extracellular calcium and calcium stored in the sarcoplasmic reticulum (SR). The amount of SR-calcium storage and the release of calcium are regulated by the $\mathrm{Ca}^{2+}$ ATPase, SERCA2a and ryanodine receptors (RyR2). A previous study showed that substrate stiffness regulates the L-type voltage-gated $\mathrm{Ca}^{2+}$ channel on embryonic chick cardiomyocytes. The peak current density of the L-type $\mathrm{Ca}^{2+}$ channel is greater on stiff PDMS substrate $(\approx 450 \mathrm{kPa})$ compared to softer substrates $(\approx 46 \mathrm{kPa})$. Indeed, the authors demonstrated that on stiff poly-Dlysine-coated substrate there is a downregulation of the expression of $\mathrm{Ca}^{2+}$-sensitive $\mathrm{K}^{+}$(BK) channels, that play a critical role in substrate stiffness regulation of the L-type $\mathrm{Ca}^{2+}$ channel [86]. Another study reported that neonatal rat ventricular myocytes (NRVMs) grown on calf skin collagen-coated acrylamide substrates, with elastic moduli ranging from 1 to $25 \mathrm{kPa}$, have a greater peak L-type $\mathrm{Ca}^{2+}$ current density, with a prolongation of the action potential duration, on $9 \mathrm{kPa}$ substrates compared to stiffer ones (16-25 kPa) [80]. Similarly, studies performed on hiPSC-CMs and NVRMs showed that, during a twitch, intracellular calcium levels increase on substrates with a stiffness in the range of that of native myocardium $[76,78]$. These studies quantified the intracellular calcium levels using Fura-2 or Fluo-4 calcium indicators, reporting a greater calcium transient amplitude in cardiomyocytes cultured on substrates with physiological values of stiffness. For instance, Rodriguez and collaborators [77] reported that maximal intracellular calcium level is reached on the stiffest fibronectin-coated substrates $(15 \mathrm{kPa})$ compared to the softest ones ( $3 \mathrm{kPa}$ ). Also, as assessed by western blot analysis, the expression of SERCA2a and RyR2 is higher in cardiomyocytes cultured on Collagen I-coated PA gels with stiffness of native myocardium [76]. Interestingly, Martewicz and collaborators identified the involvement of the RhoA/ROCK pathway in the structural/functional changes of hiPSC-CMs after adhesion plating onto stiffer laminin/fibronectin-coated substrates (4 kPa $v s 16 \mathrm{kPa} v s$ glass). The process is triggered by cell interaction with the stiffer substrate and is associated with re-localization of SERCA2 pumps from a perinuclear to a whole-cell distribution with concurrent improvement SR calcium buffering [87].

\subsection{Force and contractile properties}

During cardiac development, mechanical loading and electrical activity represent two major factors for cardiomyocyte growth and maturation. Studies have shown that hiPSC-CMs used to create collagen I-based engineered heart tissues (EHTs) were subjected to different mechanical stress and electrical pacing conditions. Ruan and collaborators [88] demonstrated that, in these conditions, the tissue had an additional increase in contractility and expression of calcium handling proteins without changes in cell size or alignment, suggesting maturation of excitation-contraction coupling. Moreover, Rodriguez et al. [77] showed that substrates with stiffness values similar to those of healthy myocardium lead to improved myofibril structure that contributes to enhance cardiomyocyte contractility, thus generating slower but more forceful twitches. Also, when combined with cell anisotropy, substrates with more physiological stiffness values allow cardiomyocytes to exhibit higher myofibril organization, cytoskeletal structure and expression of maturation markers [78]. Lastly, although little is reported in the literature, we and other groups reported benefits of myofibril organization and force production from patterned substrates with rectangular micro/nanogrooved geometry [81,89-92]. For instance, Ribeiro and coworkers compared the expression of cTnI, cardiac Troponin $\mathrm{T}(\mathrm{cTnT})$, and titin isoforms (N2A and N2B) in hiPSC-CMs cultured both on unpatterned PA substrates and PA hydrogels with rectangular Matrigel patterns with a Young's modulus of $6-35 \mathrm{kPa}$. Their results support the idea that both substrate stiffness and cell shape, that is facilitated by rectangular microgrooves in polyacrylamide substrates, tune the sarcomere activity and the mechanical output of hiPSC-CMs [81]. Moreover, Weber and colleagues [93] found more abundancy of $\beta$ - versus $\alpha$ - myosin heavy chain (MHC) on laminin coated- (i.e., a stiffer substrate) versus Matrigel ${ }^{\circledR}$-coated (i.e., a softer substrate) glass, which greatly impacts the myofibril kinetics of activation and relaxation.

Importantly, one major consequence of culturing cardiomyocytes on stiff substrates is the force developed by cardiomyocytes themselves. In this context, as indicated in Table 1 , several studies support the idea that hiPSC-CMs, embryonic chick cardiomyocytes, NRVMs can adapt their contractile properties on scaffolds with physical properties typical of native myocardium.

In agreement with the obseravations of Martewicz and cowerkers [87], Jacot and coworkers report that substrate stiffness affects cardiomyocyte contractility through involvement of the RhoA/RhoAkinase (ROCK) pathway that acts to prevent actin depolymerization and inhibits myosin light chain phosphatase. The latter events may otherwise result in an increase of FAs and development of stress fibers that have a negative effect on sarcomere formation. Thus, compared to environments with stiffness value near that of native myocardium, stiffer substrates, in which ROCK pathway is activate, prevent sarcomere development and cardiomyocyte maturation [76]. In Fig. 3A, we compared different studies that evaluated cardiomyocyte tension over a softer substrate stiffness (4 to $20 \mathrm{kPa}$ ). Despite some variability, force production seems to be directly related to increased substrate stiffness regardless of the type of cardiomyocyte evaluated (hiPSC-CMs or NRVMs) [77,81,91,94]. Nevertheless, other studies performed on embryonic chick cardiomyocytes and hiPSC-CMs support the hypothesis that performing cell culture on substrates with stiffness near that of myocardium can lead to higher force generation. In Fig. 3B, we plotted values of cardiomyocyte tension over a more rigid substrate stiffness (20 to $100 \mathrm{kPa}$ ) modified from different studies [91,95-97]. Tension of hiPSC-CMs was shown to increase when cultured on substrates of up to $90 \mathrm{kPa}$, reaching its maximum value, above which no further increases were observed [91]. A possible explanation is shown in Fig. 3C-D in which we considered two studies that resolved cell or sarcomere shortening related to force generation [91,95]. The upper panels are modified charts from Hazeltine et al. [95] and Ribeiro et al. [91] showing cell shortening related to substrate stiffness. For instance, Ribeiro and coworkers demonstrated that both cell and sarcomere shortening are not affected beyond physiological levels of stiffness (21 $\mathrm{kPa}$ ) in hiPSC-CMs cultured on gelatin-coated PA gels. In the lower panels, we plotted the tension values against the relative cell shortening of both studies. In both cases, the curve decreases indicating that, with increasing Young's modulus, cell shortening is very low, however tension generation gets higher until a plateau phase. It suggests that cardiomyocytes are subjected to greater resistance when pulling on stiffer substrates. This can be related to increased external load due to a stronger bound between integrins and ECM proteins compared to the softer substrates, likely resembling a more isometric condition. One possible explanation of this behaviour is that cardiomyocytes are 
A

\section{Softer substrates}

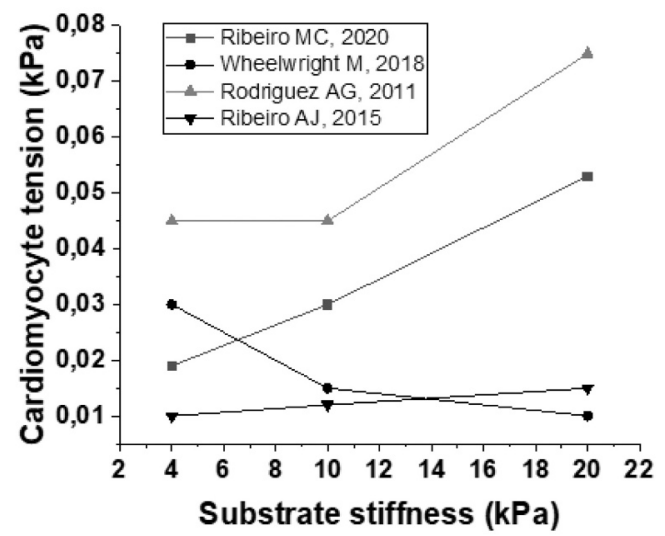

C
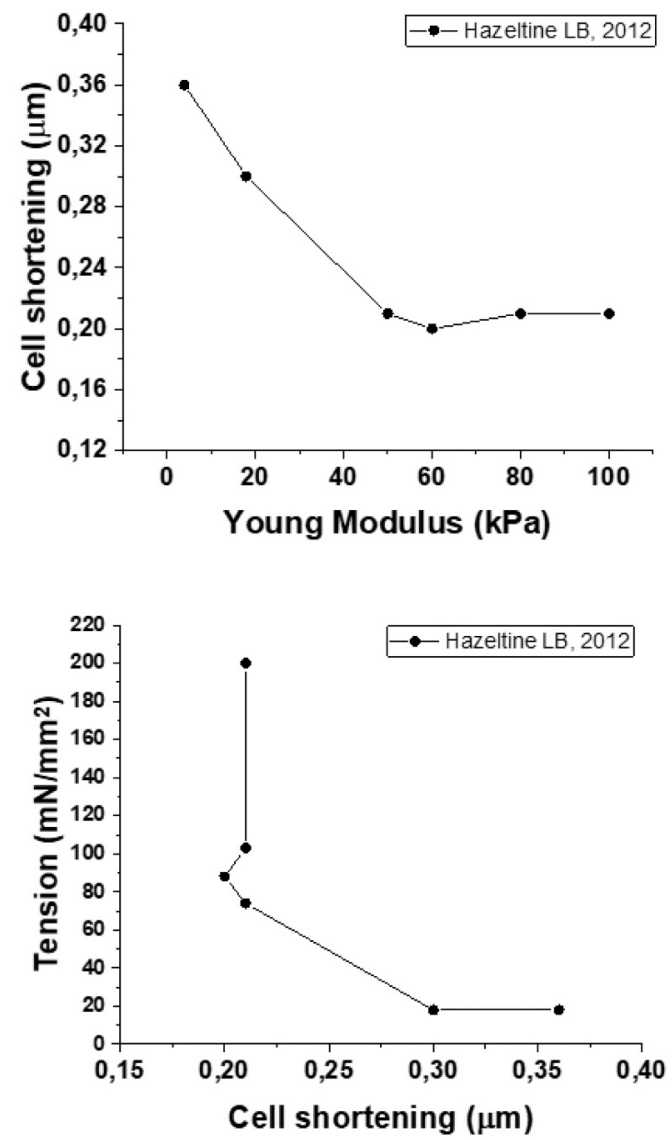

B

\section{Rigid substrates}

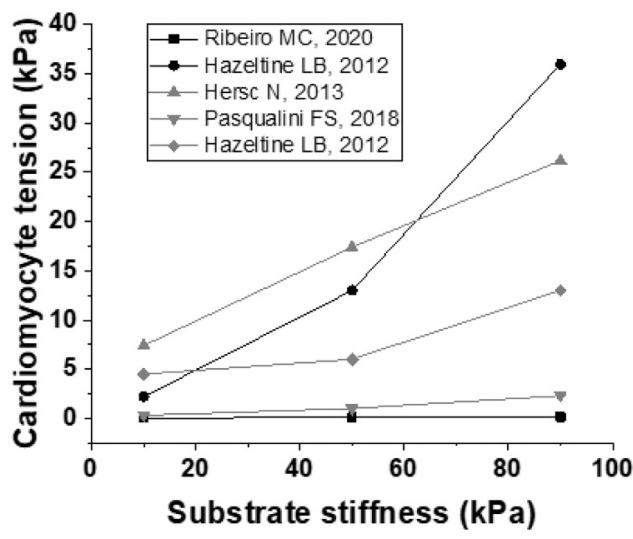

D
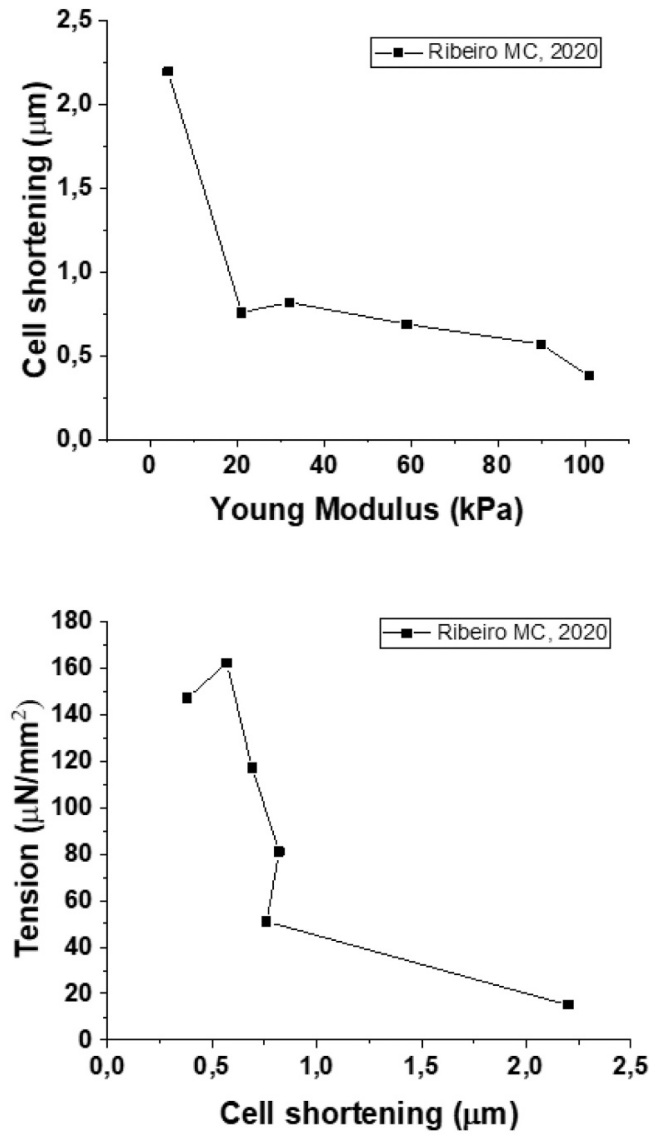

Fig. 3. How substrate stiffness affects cardiomyocyte force production. Studies reporting single cardiomyocytes (CMs) were selected on the basis of substrate stiffness using measurements obtained with traction tests or microposts arrays involving hiPSC-CMs (black) or NRMVs (grey). CM tension production was reported as kPa (1 $\mathrm{kPa}=1 \mathrm{mN} / \mathrm{mm}^{2}$ ). (A) CM tension was related to softer substrates, with similar range of physiological stiffness values (Young's Moduli 4-10-20 kPa), (B) and to a wider range of more rigid substrates (Young's Moduli 10-50-90 kPa). (C) A modified plot analysis from Hazeltine LB et al., 2012 [95] showing traction force microscopy. Cell shortening $(\mu \mathrm{m})$ is plotted against substrate stiffness (Young's Modulus, $\mathrm{kPa}$ ) and $\mathrm{CM}$ tension $\left(\mathrm{mN} / \mathrm{mm}^{2}\right.$ ) versus cell shortening. (D) Similarly, a modified plot analysis from Ribeiro MC et al. 2020 [91]. 
anchored in culture through FAs [97]. These specialized sites, that link cECM to cardiomyocyte cytoskeleton via integrin receptors, participate in cell force transmission to the substrate. Compared to stiffer substrates, on softer scaffolds cardiomyocytes cannot form as many FAs due to lower actomyosin contractions and more immature aggregation of integrins. Consistently, lower force production is observed on the softest gels since a smaller amount of force is required to detach the cells from the substrate and cardiomyocytes are not able to generate isometric tension.

Nevertheless, in this context little is still known on the contraction kinetics related to substrate stiffness and the assumptions on the possible mechanisms involved are elusive. For instance, as reported by Ribeiro et al. [91], the velocity of contraction and relaxation of hiPSCs significantly decreases from $4 \mathrm{kPa}$ up to $32 \mathrm{kPa}$ and then it is kept constant at higher substrate stiffness. Thus, the speed of contraction and relaxation does not adapt to enhanced stiffness beyond the physiological-like stiffness values. In addition, a work performed by the van der Velden group [98] reports the impact of matrix stiffness on adult rat ventricular cardiomyocytes (ARVMs) measured after detachment of cardiomyocytes from the underlying surface. They demonstrated that matrix stiffening impairs the velocity of contraction and relaxation as well as cell contraction, indicating that $\mathrm{Ca}^{2+}$ reuptake is lower in these conditions.

Overall, as these measurements are highly dependent on the experimental conditions and model, the comparison and understanding of absolute stiffness values still constitute a major limitation in this field of research. Therefore, contrasting results are related, no doubt, to the diversity of cardiomyocytes used. Most studies investigated the impact of matrix stiffening on stem cell-derived, embryonic, or neonatal cardiomyocytes. Compared to adult cardiomyocytes, these more immature cells are less challenging to maintain in culture and relatively easy to be isolated. Their major disadvantage relies, though, on the immature features. On the other hand, this limitation is also considered particularly useful because of the marked cellular plasticity to adapt to a different microenvironment.

\section{In vitro applications of substrates with tunable stiffness}

Although substrate stiffness has been previously described or reviewed [3,99-101], further advances made this tool more intriguing. In particular, the combination of biomaterials with known elastic modulus and hiPSC-CMs may open new perspectives as in vitro models for studying cECM-cell interaction. Firstly, the fact that hiPSC-CMs can adapt to elastic properties of the underlying substrate raises some questions on the involvement of cECM-cell interaction during cardiac development. Secondly, hiPSC-CM models obtained from patients with mutations in genes encoding for protein complexes of the costamere may similarly help to elucidate this aspect. Many of the genes encoding for proteins involved in the cECM-cell mechanosignalling are often associated with dilated cardiomyopathies (DCM) [102,103] and hypertrophic cardiomyopathies (HCM) [104].

\subsection{Advances of substrate stiffness for hiPSC-CM maturation}

The composition, architecture and mechanical properties of cardiac extracellular microenvironment are subject to changes during development, instructing resident cells and guiding them toward maturation [44]. It is well known that hiPSC-CMs are generally characterized by an immature phenotype that is still far from recapitulating all the aspects of healthy and diseased human adult cardiomyocytes. Thus, the attempt of obtaining hiPSC-CMs with more mature structure and function is a primary goal in this field. With this purpose, a limiting factor is constituted by $2 \mathrm{D}$ in vitro cultures that widely differs from the $3 \mathrm{D}$ environment of a whole heart. Most of the differences are related to spontaneous beating, cell flattening and cell spreading, which are typical features of immature cardiomyocytes and thus not observed in adult cells. Therefore, several strategies have been investigated to enhance maturity of hiPSC-CMs in vitro, including long-term culture, mechanical or electrical stimulation, co-culture with non-myocytes, three-dimensional (3D) constructs and use of specific materials as cell scaffolds [105]. As already mentioned, several studies suggest a major impact of substrates with Young's modulus near to that of healthy myocardium (\&10-20 kPa) [76,83,106]. However, other studies reported a greater force production on apparently non-physiological stiffness values $[85,95,107]$. Taken together, these studies agree on a linear correlation between substrate stiffness and increased contractility and myofibrillar organization.

To better understand this relationship, a major focus should be on identification of cellular stretching elements sensitive to substrate stiffness. For instance, the lipid composition of the sarcolemma may affect cell rigidity $[108,109]$ as well as mechano-gated ion channels of the TRP family, stretch-activated channels (SACs, Piezos, TREKs) or polycystines 2 (PC2), that interfere with calcium dynamics [110-112], and integrins [61]. Not least, the actin cytoskeleton and its interacting proteins, such as DGC proteins (dystrophin) or filamin, have been shown to be essential for these interactions. Many of these multimeric protein complexes are also important for integrity of cell structure, transmission of force as well as mechanosensing and mechanotransduction.

This issue is of crucial relevance for the design of in vitro cardiac platforms, which aim to recapitulate specific aspects of cECM-cell interaction. Engineering biomaterials to mimic the native $\mathrm{CECM}$ is still challenging due to the high complexity of the cECM environment. Specifically, biomaterials need to meet essential criteria such as: longterm elasticity, adaptable biodegradability, biocompatibility, outsidein-signalling and tunable mechanical properties. The most broadly used biomaterials for cardiac scaffolds are hydrogels that have useful biochemical and biophysical properties. Among the natural hydrogel substrates, the use of collagen I, fibrin, vitronectin, gelatin, hyaluronic acid, alginate and Matrigel has been investigated. However, their limited availability, high costs and batch-to-batch variation represent important drawbacks of natural materials. Therefore, synthetic hydrogels have also been explored, although they have reduced biocompatibility and could produce adverse reactions. Among them, there are polyesters, poly-lactones, elastomers, such as polyurethane (PU) and poly-ethylene glycol (PEG), that are degradable and have unlimited availability and tunable mechanical properties [15]. The application of hydrogels requires a casting mold to obtain the desired form and, in addition to the mold, anchoring supports are needed to provide the mechanical loading required for tissue remodelling along with cardiomyocyte maturation and alignment [113]. In the field of cardiac tissue engineering, another possible strategy provides for the use of decellularized CECM as a scaffold with intact 3D structure. These strategies represent the most traditional approaches to generate engineered heart tissues (EHTs). Thanks to the more sophisticated techniques now available, such as nanomaterial technology and $3 \mathrm{D}$ printing, it is possible to design more complex microenvironments characterized by precisely controlled dimensions and geometries, mimicking the native myocardium. For instance, there is a growing interest in employing materials that can dynamically change their properties in the presence of cells to study mechanobiology [114]. A crucial aspect that is generally not considered is that conventional $2 \mathrm{D}$ and $3 \mathrm{D}$ cultures are static substrates with specific Young's Modulus. These conditions are far from recapitulating the dynamic process of tissue stiffening that naturally occurs in the native myocardium in both physiological and pathological conditions. Using static substrates, the adaptation of cardiomyocytes to a specific matrix stiffness may reflect a mechanical response of the cells to a condition that does not necessarily recapitulate all the phases occurring during in vivo cECM remodelling. This is a major limitation for mimicking the dynamic process that ultimately leads to cECM remodelling and changes at the cellular level. Therefore, a major step forward may include the use of materials that can change their stiffness along the time in culture. For instance, dual-stage polymerization reactions represent an innovative tool for recreating heterogeneous and localized 
ECM stiffening in vitro, thus recreating the dynamic ECM remodelling $[115,116]$.

Finally, in the field of cardiac regenerative strategies, liquid crystalline elastomers (LCEs) have shown to be promising smart materials to assist cardiac contraction, on the macroscale, and to support cell growth, on the microscale. Importantly, Martella et al. demonstrated that these polymers are biocompatible, as assessed by preliminary experiments on murine myoblasts (C2C12 cell line), human dermal fibroblasts and hiPSC-CMs [117]. Also, LCEs have the unique property to be activated and to autonomously contract upon application of various external stimuli, opening the possibility to synchronize the contraction of these polymers with that of cardiac cells such as hiPSC-CMs [118]. For instance, the Verduzco group demonstrated that LCEs can exhibit reversible shape changes in response to cyclic heating and electrical stimulation thus allowing to be exploited as responsive substrates to culture cardiomyocytes [119]. Therefore, while common biomaterials provide just with their elastic properties to the substrate by interacting with cells, dynamic cell scaffolds as LCEs can mimic the cycles of cardiac contraction, ultimately giving an additional active mechanical input to the attached cardiomyocytes. In summary, cardiomyocytes natively exist within a 3D microenvironment. Overall, future studies should address whether 3D cell culture systems (e.g. EHTs, decellularized myocardium or biomaterial-based scaffold) involving variable stiffness can yield similar findings as the $2 \mathrm{D}$ studies. The protein composition, imposed geometry, dynamic stiffening and load of the 3D extracellular matrix are overall conditions that need to be considered for mimicking more closely the native cardiac microenvironment.

\subsection{Impact of substrate stiffness on in vitro cardiomyopathy modelling}

Since the cECM is a living reactive structure, it undergoes continuous remodelling, in a delicate balance between physiological and pathological processes [120]. Altered expression of cECM components or changes in downstream signalling pathways can lead to cardiac impairments that resemble those found in patients with congenital heart diseases [121]. Several genetic cardiomyopathies are characterized by cardiac fibrosis which, on a molecular scale, is indicated as an increase in cardiac tissue stiffness [5]. In this context, hiPSC-CMs obtained from patients are the most suitable platform for the investigation of earlystage defects related to the onset of many cardiomyopathies. To this aim, the possibility of targeting genes of interest by CRISPR-Cas9 gene editing technology allows the validation of the pathogenicity of any cardiomyopathy-associated variant $[60,122]$. Moreover, it may contribute to elucidate the role of specific proteins with debated function and involved in physiological responses during cECM stiffening. For instance, a previous report showed the impact of soft and stiff substrates to study the importance of dystrophin in hiPSC lines obtained from Duchenne Muscular Dystrophy (DMD) patients. Chang et al. purposed the idea that tissue stiffness may trigger mechanisms related to contractile dysfunction, oxidative stress and telomere shortening in DMD cardiomyocytes [123]. Indeed, the complexity of DGC-associated proteins is finely regulated and dysregulation of their downstream pathways has fatal consequences. For instance, conditional deletion of a DGC-associated protein called agrin is sufficient to compromise cardiac regeneration and function [124]. Agrin is a widely expressed proteoglycan that interacts with its receptor $\alpha$-dystroglycan leading to disassembly of the DCG complex. This event enables Yap translocation to the nucleus where it promotes cardiomyocyte proliferation. The dysregulation of the hippo pathway is associated with cardiac hypertrophy, DCM and ischemic cardiac disease [71].

In addition, the impact of tissue stiffness and fibrosis can be better elucidated in the 3D format using EHTs [125,126]. A report from Sewanan and collaborators analyzed the effect of decellularized porcine myocardium with the HCM R403Q-MYH7 mutation on healthy hiPSCCMs. The mutant HCM-ECM was about $2.7 \times$ stiffer than healthy ECM. Surprisingly, hiPSC-CMs encapsulated in HCM-ECM showed both increased amplitude of calcium transient and force production compared to those on healthy ECM [127], in a similar way to what is reported for substrate stiffness. However, a possible explanation of the underlying mechanisms cannot be limited to a mere mechanical effect. Indeed, stiffer substrates were associated with hypertrophic signalling, suggesting that in this context the use of biomaterials may help to better understand how cECM contributes to cardiac hypertrophy [128]. Notably, the application of an external load was tested in EHT using hiPSC-CMs. Increased levels of afterload lead to improved calcium handling and expression of several cardiac maturation markers (i.e., a shift from $\alpha$ to $\beta$ myosin heavy chain isoform predominance) [93]. However, the highest afterload condition (continuous isometric contraction), revealed markers of pathological hypertrophy and fibrosis [85]. Overall, the possibility of tuning the mechanical properties of biomaterials for mimicking in vitro cECM has a crucial relevance in recapitulating pathophysiological microenvironments and triggering signalling pathways determining cell phenotype and function. Further advances may include the recent generation of a tissue-engineered scale models of human myocardium made of nanofibrous scaffolds. They have been used to study pressure-volume loops in presence or absence of test compounds similarly to assays performed on animal ventricular or atrial tissue [129]. Finally, the opportunity to combine hiPSC-CMs, endothelial cells, and fibroblasts [130] with biomaterials paves the way for identifying specific mechanisms involved in cardiac diseases that may be relevant to unveil the pathogenesis of many cardiomyopathies.

\section{Conclusions}

This review discusses potential mechanisms that may explain the influence of substrate stiffness on cardiomyocyte properties. In Fig. 4, the main biomechanical effects of substrate stiffness on in vitro cardiac models are summarized. Increased force in cardiomyocytes cultured on stiffer matrices might be related with a tight anchorage of cardiomyocytes to the substrate, imparting higher load, more isometric contraction and activation of mechano-signalling pathways related to cell hypertrophy (e.g., YAP/TAZ) [3,131,132]. Better understanding of the impact of substrate stiffness on hiPSC-CM function will provide new insights to create biomaterials that may recapitulate the ECM alterations occurring in several pathologies (e.g., cardiomyopathies, dystrophies). For this reason, this work encourages synergy between the biomaterial and cardiac muscle community. Future investigations using hiPSC-CM models will promote the interpretability of defects in cECM-cell interaction for targeting cECM with specific compounds before the advent of myocardial stiffening and fibrosis associated with inherited cardiomyopathies. For this purpose, the advances of dynamic smart materials with tuneable stiffness may represent the ideal tool to unveil the mechanisms underlying cECM remodelling in genetic cardiomyopathies.

\section{Funding}

This work is supported by a Telethon Italy Grant (GUP19012B, C.F. and G.P.). The first author of this review, Silvia Querceto, is supported by a doctoral fellowship (Pegaso, Regione Toscana) from the University of Siena. A.G. is supported by the European Commission ERA-NET on Cardiovascular Diseases JTC2018 "Transnational Cardio-vascular Research Projects driven by Early Career Scientists", JTC2018-046 DENIM. Any opinions and conclusions or recommendations expressed in this material are those of the authors and do not necessarily reflect the views of the funding sources.

\section{Author contributions}

SQ, CF, and JMP contributed to the design and wrote the manuscript in consultation with RS, AG, BG, GP, MR, CT, and CP. SQ and JMP performed the meta-analysis and prepared figures and tables. All authors critically reviewed the manuscript and declared that the research 


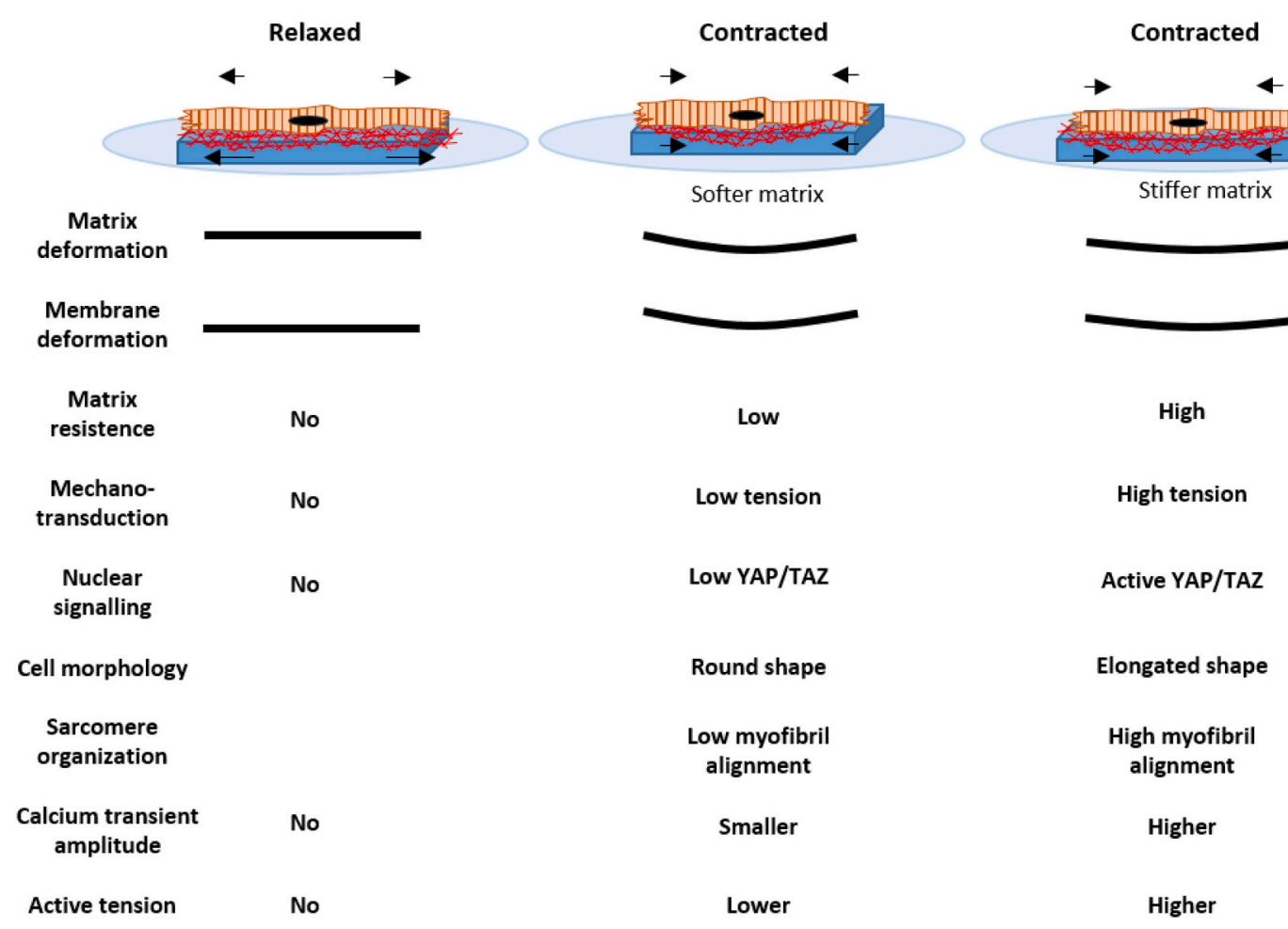

Fig. 4. Comparison between softer and stiffer matrix in relaxed and contracted state.

In cardiomyocyte (CM) relaxed state, matrix deformation, resistance and downstream mechanotransduction are null. Compared to softer substrate, CMs have higher matrix resistance, form many focal adhesions (FAs), generate higher tension and the YAP/TAZ pathways is more active.

was conducted in the absence of any commercial or financial relationships that could be construed as a potential conflict of interest.

\section{References}

[1] X. Yang, L. Pabon, C.E. Murry, Engineering adolescence: maturation of human pluripotent stem cell-derived cardiomyocytes, Circ. Res. 114 (3) (2014) 511-523.

[2] D.E. Discher, D.J. Mooney, P.W. Zandstra, Growth factors, matrices, and forces combine and control stem cells, Science 324 (5935) (2009) 1673-1677.

[3] L. Smith, S. Cho, D.E. Discher, Mechanosensing of matrix by stem cells: from matrix heterogeneity, contractility, and the nucleus in pore-migration to cardiogenesis and muscle stem cells in vivo, Semin. Cell Dev. Biol. 71 (2017) 84-98.

[4] M.L. McCain, et al., Cooperative coupling of cell-matrix and cell-cell adhesions in cardiac muscle, Proc. Natl. Acad. Sci. U. S. A. 109 (25) (2012) 9881-9886.

[5] N.G. Frangogiannis, Cardiac fibrosis, Cardiovasc. Res. 117 (6) (2021) 1450-1488.

[6] L. Marcucci, et al., Fibre and extracellular matrix contributions to passive forces in human skeletal muscles: an experimental based constitutive law for numerical modelling of the passive element in the classical hill-type three element model, PLoS One 14 (11) (2019), e0224232.

[7] T.J.M. Dick, J.M. Wakeling, Geometric models to explore mechanisms of dynamic shape change in skeletal muscle, R. Soc. Open Sci. 5 (5) (2018), 172371.

[8] M. Rienks, et al., Myocardial extracellular matrix: an ever-changing and diverse entity, Circ. Res. 114 (5) (2014) 872-888.

[9] P. Camelliti, T.K. Borg, P. Kohl, Structural and functional characterisation of cardiac fibroblasts, Cardiovasc. Res. 65 (1) (2005) 40-51.

[10] J.R. Gershlak, et al., Mesenchymal stem cells ability to generate traction stress in response to substrate stiffness is modulated by the changing extracellular matrix composition of the heart during development, Biochem. Biophys. Res. Commun. 439 (2) (2013) 161-166.

[11] E.A. Castillo, K.V. Lane, B.L. Pruitt, Micromechanobiology: focusing on the cardiac cell-substrate Interface, Annu. Rev. Biomed. Eng. 22 (2020) 257-284.

[12] B. Speiser, C.F. Riess, J. Schaper, The extracellular matrix in human myocardium: part I: collagens I, III, IV, and VI, Cardioscience 2 (4) (1991) 225-232.

[13] T.K. Borg, R.E. Gay, L.D. Johnson, Changes in the distribution of fibronectin and collagen during development of the neonatal rat heart, Coll. Relat. Res. 2 (3) (1982) 211-218.

[14] H. Kim, et al., Expression of extracellular matrix components fibronectin and laminin in the human fetal heart, Cell Struct. Funct. 24 (1) (1999) 19-26.

[15] V. Schwach, R. Passier, Native cardiac environment and its impact on engineering cardiac tissue, Biomater. Sci. 7 (9) (2019) 3566-3580.

[16] M.D. Combs, K.E. Yutzey, Heart valve development: regulatory networks in development and disease, Circ. Res. 105 (5) (2009) 408-421.
[17] A.C. Gittenberger-de Groot, et al., Collagen type VI expression during cardiac development and in human fetuses with trisomy 21, Anat Rec A Discov Mol Cell Evol Biol 275 (2) (2003) 1109-1116.

[18] H. Mollnau, B. Munkel, J. Schaper, Collagen VI in the extracellular matrix of normal and failing human myocardium, Herz 20 (2) (1995) 89-94.

[19] S.E. Klewer, et al., Expression of type VI collagen in the developing mouse heart, Dev. Dyn. 211 (3) (1998) 248-255.

[20] C.F. Guimarães, et al., The stiffness of living tissues and its implications for tissue engineering, Nat. Rev. Mat. 5 (5) (2020) 351-370.

[21] K. Reiser, R.J. McCormick, R.B. Rucker, Enzymatic and nonenzymatic crosslinking of collagen and elastin, FASEB J. 6 (7) (1992) 2439-2449.

[22] M. Yamauchi, M. Terajima, M. Shiiba, Lysine hydroxylation and cross-linking of collagen, Methods Mol. Biol. 1934 (2019) 309-324.

[23] H.L. Granzier, T.C. Irving, Passive tension in cardiac muscle: contribution of collagen, titin, microtubules, and intermediate filaments, Biophys. J. 68 (3) (1995) 1027-1044.

[24] B. López, et al., Impact of treatment on myocardial lysyl oxidase expression and collagen cross-linking in patients with heart failure, Hypertension 53 (2) (2009) 236-242.

[25] B. López, et al., Role of lysyl oxidase in myocardial fibrosis: from basic science to clinical aspects, Am. J. Physiol. Heart Circ. Physiol. 299 (1) (2010) H1-H9.

[26] W.A. Linke, Sense and stretchability: the role of titin and titin-associated proteins in myocardial stress-sensing and mechanical dysfunction, Cardiovasc. Res. 77 (4) (2008) 637-648.

[27] W.A. Linke, Titin gene and Protein functions in passive and active muscle, Annu. Rev. Physiol. 80 (2018) 389-411.

[28] R. Eldemire, et al., The Sarcomeric spring protein titin: biophysical properties, molecular mechanisms, and genetic mutations associated with heart failure and cardiomyopathy, Curr. Cardiol. Rep. 23 (9) (2021) 121.

[29] P.E. Marszalek, et al., Mechanical unfolding intermediates in titin modules, Nature 402 (6757) (1999) 100-103.

[30] K. Wang, et al., Regulation of skeletal muscle stiffness and elasticity by titin isoforms: a test of the segmental extension model of resting tension, Proc. Natl. Acad. Sci. U. S. A. 88 (16) (1991) 7101-7105.

[31] K. Wang, et al., Viscoelasticity of the sarcomere matrix of skeletal muscles. The titin-myosin composite filament is a dual-stage molecular spring, Biophys. J. 64 (4) (1993) 1161-1177.

[32] S. Dutta, et al., Calcium increases titin N2A binding to F-actin and regulated thin filaments, Sci. Rep. 8 (1) (2018) 14575.

[33] R.J. van der Pijl, C.A.C. Ottenheijm, Titin N2A: more than a signaling node? J. Gen. Physiol. 153 (7) (2021).

[34] M.L. Bang, et al., The complete gene sequence of titin, expression of an unusual approximately $700-\mathrm{kDa}$ titin isoform, and its interaction with obscurin identify a novel Z-line to I-band linking system, Circ. Res. 89 (11) (2001) 1065-1072. 
[35] M.L. Greaser, et al., Developmental changes in rat cardiac titin/connectin: transitions in normal animals and in mutants with a delayed pattern of isoform transition, J. Muscle Res. Cell Motil. 26 (6-8) (2005) 325-332.

[36] K. Watanabe, et al., Molecular mechanics of cardiac titin's PEVK and N2B spring elements, J. Biol. Chem. 277 (13) (2002) 11549-11558.

[37] C. Hidalgo, H. Granzier, Tuning the molecular giant titin through phosphorylation: role in health and disease, Trends Cardiovasc. Med. 23 (5) (2013) 165-171.

[38] J.A. Rivas-Pardo, et al., Work done by titin protein folding assists muscle contraction, Cell Rep. 14 (6) (2016) 1339-1347.

[39] N. Fukuda, H.L. Granzier, Titin/connectin-based modulation of the frank-Starling mechanism of the heart, J. Muscle Res. Cell Motil. 26 (6-8) (2005) 319-323.

[40] H. Tsutsui, K. Ishihara, G. Cooper, Cytoskeletal role in the contractile dysfunction of hypertrophied myocardium, Science 260 (5108) (1993) 682-687.

[41] P. Robison, et al., Detyrosinated microtubules buckle and bear load in contracting cardiomyocytes, Science 352 (6284) (2016) aaf0659.

[42] C.Y. Chen, et al., Suppression of detyrosinated microtubules improves cardiomyocyte function in human heart failure, Nat. Med. 24 (8) (2018) 1225-1233.

[43] M. Takaza, et al., The anisotropic mechanical behaviour of passive skeletal muscle tissue subjected to large tensile strain, J. Mech. Behav. Biomed. Mater. 17 (2013) 209-220.

[44] M. Ward, T. Iskratsch, Mix and (mis-)match - the mechanosensing machinery in the changing environment of the developing, healthy adult and diseased heart, Biochim. Biophys. Acta, Mol. Cell Res. 1867 (3) (2020), 118436.

[45] M. Lockhart, et al., Extracellular matrix and heart development, Birth Defects Res. A Clin. Mol. Teratol. 91 (6) (2011) 535-550.

[46] M. Kruger, W.A. Linke, Titin-based mechanical signalling in normal and failing myocardium, J. Mol. Cell. Cardiol. 46 (4) (2009) 490-498.

[47] S. Lahmers, et al., Developmental control of titin isoform expression and passive stiffness in fetal and neonatal myocardium, Circ. Res. 94 (4) (2004) 505-513.

[48] C.A. Opitz, et al., Developmentally regulated switching of titin size alters myofibrillar stiffness in the perinatal heart, Circ. Res. 94 (7) (2004) 967-975.

[49] C.M. Warren, et al., Titin isoform changes in rat myocardium during development, Mech. Dev. 121 (11) (2004) 1301-1312.

[50] Y. Tan, et al., Probing the mechanobiological properties of human embryonic stem cells in cardiac differentiation by optical tweezers, J. Biomech. 45 (1) (2012) $123-128$.

[51] W. Kwiecinski, et al., Cardiac shear-wave elastography using a transesophageal transducer: application to the mapping of thermal lesions in ultrasound transesophageal cardiac ablation, Phys. Med. Biol. 60 (20) (2015) 7829-7846.

[52] A. Kolipaka, et al., Magnetic resonance elastography as a method for the assessment of effective myocardial stiffness throughout the cardiac cycle, Magn. Reson. Med. 64 (3) (2010) 862-870.

[53] K.H. Nakayama, L. Hou, N.F. Huang, Role of extracellular matrix signaling cues in modulating cell fate commitment for cardiovascular tissue engineering, Adv Healthc Mater 3 (5) (2014) 628-641.

[54] J.V. Pardo, J.D. Siliciano, S.W. Craig, A vinculin-containing cortical lattice in skeletal muscle: transverse lattice elements ("costameres") mark sites of attachment between myofibrils and sarcolemma, Proc. Natl. Acad. Sci. U. S. A. 80 (4) (1983) 1008-1012

[55] S.W. Craig, J.V. Pardo, Gamma actin, spectrin, and intermediate filament proteins colocalize with vinculin at costameres, myofibril-to-sarcolemma attachment sites, Cell Motil. 3 (5-6) (1983) 449-462.

[56] J.M. Ervasti, Costameres: the Achilles' heel of herculean muscle, J. Biol. Chem. 278 (16) (2003) 13591-13594.

[57] T. Voelkel, W.A. Linke, Conformation-regulated mechanosensory control via titin domains in cardiac muscle, Pflugers Arch. 462 (1) (2011) 143-154.

[58] M. Gautel, Cytoskeletal protein kinases: titin and its relations in mechanosensing, Pflugers Arch. 462 (1) (2011) 119-134.

[59] A.K. Peter, et al., The costamere bridges sarcomeres to the sarcolemma in striated muscle, Prog. Pediatr. Cardiol. 31 (2) (2011) 83-88.

[60] J.M. Pioner, et al., Advances in stem cell modeling of dystrophin-associated disease: implications for the wider world of dilated cardiomyopathy, Front Physiol. 11 (2020) 368.

[61] R. Santoro, et al., Unchain my heart: Integrins at the basis of iPSC cardiomyocyte differentiation, Stem Cells Int. 2019 (2019) 8203950.

[62] M. Brancaccio, et al., Differential onset of expression of alpha 7 and beta 1D integrins during mouse heart and skeletal muscle development, Cell Adhes. Commun. 5 (3) (1998) 193-205.

[63] A.M. Wiencierz, et al., Differential expression levels of integrin alpha6 enable the selective identification and isolation of atrial and ventricular cardiomyocytes, PLoS One 10 (11) (2015), e0143538.

[64] A.S. Torsoni, et al., Focal adhesion kinase is activated and mediates the early hypertrophic response to stretch in cardiac myocytes, Circ. Res. 93 (2) (2003) $140-147$.

[65] A.P. Dalla Costa, et al., FAK mediates the activation of cardiac fibroblasts induced by mechanical stress through regulation of the mTOR complex, Cardiovasc. Res. 86 (3) (2010) 421-431.

[66] K.T. Weber, et al., Myofibroblast-mediated mechanisms of pathological remodelling of the heart, Nat. Rev. Cardiol. 10 (1) (2013) 15-26.

[67] T.J. Herron, et al., Extracellular matrix-mediated maturation of human pluripotent stem cell-derived cardiac monolayer structure and electrophysiological function, Circ. Arrhythm. Electrophysiol. 9 (4) (2016), e003638.
[68] K. Burridge, K. Wennerberg, Rho and Rac take center stage, Cell 116 (2) (2004) 167-179.

[69] B.R. Freedman, et al., The (dys)functional extracellular matrix, Biochim. Biophys. Acta 1853 (11 Pt B) (2015) 3153-3164.

[70] R. Santoro, et al., Activation of human aortic valve interstitial cells by local stiffness involves YAP-dependent transcriptional signaling, Biomaterials 181 (2018) 268-279.

[71] M.M. Mia, M.K. Singh, The hippo signaling pathway in cardiac development and diseases, Front. Cell. Dev. Biol. 7 (2019) 211.

[72] Q. Zhou, et al., The hippo pathway in heart development, regeneration, and diseases, Circ. Res. 116 (8) (2015) 1431-1447.

[73] M. Aragona, et al., A mechanical checkpoint controls multicellular growth through YAP/TAZ regulation by actin-processing factors, Cell 154 (5) (2013) 1047-1059.

[74] G. Forte, et al., Substrate stiffness modulates gene expression and phenotype in neonatal cardiomyocytes in vitro, Tissue Eng. Part A 18 (17-18) (2012) $1837-1848$.

[75] P. Pandey, et al., Cardiomyocytes sense matrix rigidity through a combination of muscle and non-muscle myosin contractions, Dev. Cell 45 (5) (2018) 661.

[76] J.G. Jacot, A.D. McCulloch, J.H. Omens, Substrate stiffness affects the functional maturation of neonatal rat ventricular myocytes, Biophys. J. 95 (7) (2008) 3479-3487.

[77] A.G. Rodriguez, et al., Substrate stiffness increases twitch power of neonatal cardiomyocytes in correlation with changes in myofibril structure and intracellular calcium, Biophys. J. 101 (10) (2011) 2455-2464.

[78] M.L. Rodriguez, et al., Substrate stiffness, cell anisotropy, and cell-cell contact contribute to enhanced structural and calcium handling properties of human embryonic stem cell-derived cardiomyocytes, ACS Biomater Sci. Eng. 5 (8) (2019) 3876-3888.

[79] P. Bajaj, et al., Stiffness of the substrate influences the phenotype of embryonic chicken cardiac myocytes, J. Biomed. Mater. Res. A 95 (4) (2010) 1261-1269.

[80] S.D. Boothe, et al., The effect of substrate stiffness on cardiomyocyte action potentials, Cell Biochem. Biophys. 74 (4) (2016) 527-535.

[81] A.J. Ribeiro, et al., Contractility of single cardiomyocytes differentiated from pluripotent stem cells depends on physiological shape and substrate stiffness, Proc. Natl. Acad. Sci. U. S. A. 112 (41) (2015) 12705-12710.

[82] B. Bhana, et al., Influence of substrate stiffness on the phenotype of heart cells, Biotechnol. Bioeng. 105 (6) (2010) 1148-1160.

[83] A.J. Engler, et al., Embryonic cardiomyocytes beat best on a matrix with heart like elasticity: scar-like rigidity inhibits beating, J. Cell Sci. 121 (Pt 22) (2008) 3794-3802.

[84] A. Chopra, et al., Reprogramming cardiomyocyte mechanosensing by crosstalk between integrins and hyaluronic acid receptors, J. Biomech. 45 (5) (2012) 824-831.

[85] A. Leonard, et al., Afterload promotes maturation of human induced pluripotent stem cell derived cardiomyocytes in engineered heart tissues, J. Mol. Cell. Cardiol. 118 (2018) 147-158.

[86] H. Zhao, et al., A role of BK Channel in regulation of ca, Biophys. J. 112 (7) (2017) 1406-1416.

[87] S. Martewicz, et al., Substrate and mechanotransduction influence SERCA2a localization in human pluripotent stem cell-derived cardiomyocytes affecting functional performance, Stem Cell Res. 25 (2017) 107-114.

[88] J.L. Ruan, et al., Mechanical stress conditioning and electrical stimulation promote contractility and force maturation of induced pluripotent stem cellderived human cardiac tissue, Circulation 134 (20) (2016) 1557-1567.

[89] J.M. Pioner, et al., Isolation and mechanical measurements of myofibrils from human induced pluripotent stem cell-derived cardiomyocytes, Stem Cell Rep. 6 (6) (2016) 885-896.

[90] D. Carson, et al., Nanotopography-induced structural anisotropy and sarcomere development in human cardiomyocytes derived from induced pluripotent stem cells, ACS Appl. Mater. Interfaces 8 (34) (2016) 21923-21932.

[91] M.C. Ribeiro, et al., A cardiomyocyte show of force: a fluorescent alpha-actinin reporter line sheds light on human cardiomyocyte contractility versus substrate stiffness, J. Mol. Cell. Cardiol. 141 (2020) 54-64.

[92] J.M. Pioner, et al., Optical investigation of action potential and calcium handling maturation of hiPSC-cardiomyocytes on biomimetic substrates, Int. J. Mol. Sci. 20 (15) (2019).

[93] N. Weber, et al., Stiff matrix induces switch to pure $\beta$-cardiac myosin heavy chain expression in human ESC-derived cardiomyocytes, Basic Res. Cardiol. 111 (6) (2016) 68.

[94] M. Wheelwright, et al., Investigation of human iPSC-derived cardiac myocyte functional maturation by single cell traction force microscopy, PLoS One 13 (4) (2018), e0194909.

[95] L.B. Hazeltine, et al., Effects of substrate mechanics on contractility of cardiomyocytes generated from human pluripotent stem cells, Int. J. Cell Biol. 2012 (2012), 508294

[96] F.S. Pasqualini, et al., Traction force microscopy of engineered cardiac tissues, PLoS One 13 (3) (2018), e0194706.

[97] N. Hersch, et al., The constant beat: cardiomyocytes adapt their forces by equal contraction upon environmental stiffening, Biol. Open 2 (3) (2013) 351-361.

[98] E.D. van Deel, et al., In vitro model to study the effects of matrix stiffening on ca(2 + ) handling and myofilament function in isolated adult rat cardiomyocytes, J. Physiol. 595 (14) (2017) 4597-4610.

[99] M.W. Curtis, B. Russell, Micromechanical regulation in cardiac myocytes and fibroblasts: implications for tissue remodeling, Pflugers Arch. 462 (1) (2011) 105-117. 
[100] M. Tallawi, et al., Effect of substrate mechanics on cardiomyocyte maturation and growth, Tissue Eng. Part B Rev. 21 (1) (2015) 157-165.

[101] J.G. Jacot, J.C. Martin, D.L. Hunt, Mechanobiology of cardiomyocyte development, J. Biomech. 43 (1) (2010) 93-98.

[102] F. Mazzarotto, et al., Reevaluating the genetic contribution of monogenic dilated cardiomyopathy, Circulation 141 (5) (2020) 387-398.

[103] E.M. McNally, L. Mestroni, Dilated cardiomyopathy: genetic determinants and mechanisms, Circ. Res. 121 (7) (2017) 731-748.

[104] R. Tadros, et al., Shared genetic pathways contribute to risk of hypertrophic and dilated cardiomyopathies with opposite directions of effect, Nat. Genet. 53 (2) (2021) 128-134.

[105] C.C. Veerman, et al., Immaturity of human stem-cell-derived cardiomyocytes in culture: fatal flaw or soluble problem? Stem Cells Dev. 24 (9) (2015) 1035-1052.

[106] S. Lee, et al., Contractile force generation by 3D hiPSC-derived cardiac tissues is enhanced by rapid establishment of cellular interconnection in matrix with muscle-mimicking stiffness, Biomaterials 131 (2017) 111-120.

[107] M.C. Ribeiro, et al., A cardiomyocyte show of force: a fluorescent alpha-actinin reporter line sheds light on human cardiomyocyte contractility versus substrate stiffness, J. Mol. Cell. Cardiol. 141 (2020) 54-64.

[108] S. Tanosaki, et al., Fatty acid synthesis is indispensable for survival of human pluripotent stem cells, iScience 23 (9) (2020), 101535.

[109] A. Sharma, et al., Stage-specific effects of bioactive lipids on human iPSC cardiac differentiation and cardiomyocyte proliferation, Sci. Rep. 8 (1) (2018) 6618.

[110] F. Sachs, Stretch-activated ion channels: what are they? Physiology (Bethesda) 25 (1) (2010) 50-56.

[111] A. Patel, et al., Canonical TRP channels and mechanotransduction: from physiology to disease states, Pflugers Arch. 460 (3) (2010) 571-581.

[112] K. Retailleau, F. Duprat, Polycystins and partners: proposed role in mechanosensitivity, J. Physiol. 592 (12) (2014) 2453-2471.

[113] J.E. Pomeroy, A. Helfer, N. Bursac, Biomaterializing the promise of cardiac tissue engineering, Biotechnol. Adv. (2019), https://doi.org/10.1016/j. biotechadv.2019.02.009.

[114] K.A. Gunay, et al., PEG-anthracene hydrogels as an on-demand stiffening matrix to study Mechanobiology, Angew. Chem. Int. Ed. Eng. 58 (29) (2019) 9912-9916.

[115] C.L. Petrou, et al., Clickable decellularized extracellular matrix as a new tool for building hybrid-hydrogels to model chronic fibrotic diseases in vitro, J. Mater. Chem. B 8 (31) (2020) 6814-6826.

[116] M. Guvendiren, J.A. Burdick, Stiffening hydrogels to probe short- and long-term cellular responses to dynamic mechanics, Nat. Commun. 3 (2012) 792.
[117] D. Martella, et al., Liquid crystalline networks toward regenerative medicine and tissue repair, Small 13 (46) (2017).

[118] C. Ferrantini, et al., Development of light-responsive liquid crystalline elastomers to assist cardiac contraction, Circ. Res. 124 (8) (2019) e44-e54.

[119] A. Agrawal, O. Adetiba, H. Kim, H. Chen, J. Jacot, R. Verduzco, Stimuliresponsive liquid crystal elastomers for dynamic cell culture, J. Mater. Res. 30 (4) (2015) 453-462.

[120] C.W. Chang, et al., Cardiac extracellular matrix proteomics: challenges, techniques, and clinical implications, Proteomics Clin. Appl. 10 (1) (2016) 39-50.

[121] S.F. Nagueh, et al., Altered titin expression, myocardial stiffness, and left ventricular function in patients with dilated cardiomyopathy, Circulation 110 (2) (2004) 155-162.

[122] J.R. Bhagwan, et al., Isogenic models of hypertrophic cardiomyopathy unveil differential phenotypes and mechanism-driven therapeutics, J. Mol. Cell. Cardiol. 145 (2020) 43-53.

[123] A.C.Y. Chang, et al., Increased tissue stiffness triggers contractile dysfunction and telomere shortening in dystrophic cardiomyocytes, Stem Cell Rep. 16 (9) (2021) 2169-2181, https://doi.org/10.1016/j.stemcr.2021.04.018.

[124] E. Bassat, et al., The extracellular matrix protein agrin promotes heart regeneration in mice, Nature 547 (7662) (2017) 179-184.

[125] I. Jorba, et al., In vitro methods to model cardiac Mechanobiology in health and disease, Tissue Eng. Part C Methods 27 (3) (2021) 139-151.

[126] S. Schaaf, et al., Human engineered heart tissue as a versatile tool in basic research and preclinical toxicology, PLoS One 6 (10) (2011), e26397.

[127] L.R. Sewanan, et al., Extracellular matrix from hypertrophic myocardium provokes impaired twitch dynamics in healthy cardiomyocytes, JACC Basic Transl. Sci. 4 (4) (2019) 495-505.

[128] J. Li, et al., Variation in stiffness regulates cardiac myocyte hypertrophy via signaling pathways, Can. J. Physiol. Pharmacol. 94 (11) (2016) 1178-1186.

[129] L.A. MacQueen, et al., A tissue-engineered scale model of the heart ventricle, Nat. Biomed. Eng. 2 (12) (2018) 930-941.

[130] E. Giacomelli, et al., Human-iPSC-derived cardiac stromal cells enhance maturation in 3D cardiac microtissues and reveal non-cardiomyocyte contributions to heart disease, Cell Stem Cell 26 (6) (2020) 862-879 e11.

[131] K.A. Jansen, et al., A guide to mechanobiology: Where biology and physics meet, Biochim. Biophys. Acta 1853 (11 Pt B) (2015) 3043-3052.

[132] W. Zhou, M. Zhao, How hippo signaling pathway modulates cardiovascular development and diseases, J Immunol Res 2018 (2018) 3696914. 